\title{
Derivative loss for Kirchhoff equations with non-Lipschitz nonlinear term
}

\author{
MARINA GHISI AND MASSIMO GobBINO
}

\begin{abstract}
In this paper we consider the Cauchy boundary value problem for the integro-differential equation

$$
u_{t t}-m\left(\int_{\Omega}|\nabla u|^{2} d x\right) \Delta u=0 \quad \text { in } \Omega \times[0, T)
$$

with a continuous nonlinearity $m:[0,+\infty) \rightarrow[0,+\infty)$.

It is well known that a local solution exists provided that the initial data are regular enough. The required regularity depends on the continuity modulus of $m$. In this paper we present some counterexamples in order to show that the regularity required in the existence results is sharp, at least if we want solutions with the same space regularity of initial data. In these examples we construct indeed local solutions which are regular at $t=0$, but exhibit an instantaneous (often infinite) derivative loss in the space variables.
\end{abstract}

Mathematics Subject Classification (2000): 35L70 (primary); 35L80, 35L90 (secondary).

\section{Introduction}

In this paper we consider the hyperbolic partial differential equation

$$
u_{t t}(t, x)-m\left(\int_{\Omega}|\nabla u(t, x)|^{2} d x\right) \Delta u(t, x)=0 \quad \forall(x, t) \in \Omega \times[0, T),
$$

where $\Omega \subseteq \mathbb{R}^{n}$ is an open set, $\nabla u$ and $\Delta u$ denote the gradient and the Laplacian of $u$ with respect to the space variables, and $m:[0,+\infty) \rightarrow[0,+\infty)$. Equation (1.1) is usually considered with initial conditions

$$
u(x, 0)=u_{0}(x), \quad u_{t}(x, 0)=u_{1}(x) \quad \forall x \in \Omega,
$$

and boundary conditions, for example of Dirichlet type (but the theory is more or less the same also with Neumann or periodic boundary conditions)

$$
u(x, t)=0 \quad \forall(x, t) \in \partial \Omega \times[0, T) .
$$

Received May 5, 2008; accepted in revised form January 14, 2009. 
From the mathematical point of view, (1.1) is probably the simplest example of quasilinear hyperbolic equation. From the mechanical point of view, this Cauchy boundary value problem is a model for the small transversal vibrations of an elastic string $(n=1)$ or membrane $(n=2)$. In the string context it was introduced by $\mathrm{G}$. Kirchhoff in [16].

A lot of papers have been devoted to existence of local or global solutions to (1.1), (1.2), (1.3). The results can be divided into four main families.

(A) Local existence in Sobolev spaces. If $m$ is a locally Lipschitz continuous function such that $m(\sigma) \geq v>0$ for every $\sigma \geq 0$ (strict hyperbolicity), then (1.1), (1.2), (1.3) admits a unique local solution for initial data in Sobolev spaces. This result was first proved by $\mathrm{S}$. Bernstein in the pioneering paper [3], and then extended with increasing generality by many authors. The more general statement is probably contained in A. Arosio and S. Panizzi [1] (see also the references quoted therein), where it is proved that the problem is locally well posed in the phase space

$$
V_{\beta}:=H^{\beta+1}(\Omega) \times H^{\beta}(\Omega)+\text { boundary conditions }
$$

for every $\beta \geq 1 / 2$.

(B) Global existence for analytic data. If $m$ is a continuous function such that $m(\sigma) \geq 0$ for every $\sigma \geq 0$ (weak hyperbolicity), and $u_{0}(x)$ and $u_{1}(x)$ are analytic functions, then (1.1), (1.2), (1.3) admits at least one global solution $(T=+\infty)$, which is analytic in the space variables for any time.

This result was proved with increasing generality by S. Bernstein [3], S. I. Pohozaev [20], A. Arosio and S. Spagnolo [2], P. D’Ancona and S. Spagnolo $[8,9]$.

(C) Local existence with assumptions between (A) and (B). More recently, F. Hirosawa [14] considered the continuity modulus $\omega$ of the nonlinear term $m$. He proved existence of local solutions in suitable classes of initial data, depending on $\omega$, both in the strictly hyperbolic and in the weakly hyperbolic case. From the point of view of local solutions these results represent an interpolation between the results of (A) and (B). The precise relations between $\omega$ and the regularity required on initial data are stated in Section 2. Roughly speaking, in the strictly hyperbolic case the situation is summed up by the following scheme:

$$
\begin{aligned}
\omega(\sigma)=o(1) & \rightarrow \text { analytic data, } \\
\omega(\sigma)=\sigma^{\alpha}(\text { with } \alpha \in(0,1)) & \rightarrow \text { Gevrey space } \mathcal{G}_{s}(\Omega) \text { with } s=(1-\alpha)^{-1}, \\
\omega(\sigma)=\sigma|\log \sigma| & \rightarrow C^{\infty}(\Omega) \text { (or } V_{\beta} \text { with finite derivative loss), } \\
\omega(\sigma)=\sigma & \rightarrow V_{1 / 2} \text { (with no derivative loss). }
\end{aligned}
$$


More regularity is required in the weakly hyperbolic case, as shown in the following scheme:

$$
\begin{aligned}
\omega(\sigma)=o(1) & \rightarrow \text { analytic data, } \\
\omega(\sigma)=\sigma^{\alpha}(\text { with } \alpha \in(0,1)) & \rightarrow \text { Gevrey space } \mathcal{G}_{s}(\Omega) \text { with } s=1+\alpha / 2, \\
\omega(\sigma)=\sigma & \rightarrow \text { Gevrey space } \mathcal{G}_{3 / 2}(\Omega) .
\end{aligned}
$$

(D) Global existence in special situations. Besides (B), there are four special cases in which global solutions are known to exist. We refer the interested reader to the quoted literature for the details. We just point out that in all these results the nonlinearity $m$ is assumed to be Lipschitz continuous and strictly positive.

- K. Nishihara [19] proved global existence for quasi-analytic initial data. This class of functions strictly contains the space of analytic functions, but it is strictly contained in every Gevrey class $\mathcal{G}_{s}(\Omega)$ with $s>1$.

- J. M. Greenberg and S. C. Hu [13], and then P. D'Ancona and S. Spagnolo [10] proved global existence for small initial data in Sobolev spaces in the case $\Omega=\mathbb{R}^{n}$, where dispersion plays a crucial role. Later on this dispersion-based approach was extended to external domains (see [23, 24] and the references quoted therein).

- S. I. Pohozaev [21] proved global existence for initial data in the Sobolev space $V_{1}$ in the case where $m(\sigma)=(a+b \sigma)^{-2}$, with $a>0, b>0$. In this particular case indeed equation (1.1) admits a second order invariant.

- In some recent papers R. Manfrin [17] (see also [11, 15, 18]) proved global existence in a new class of nonregular initial data. Manfrin's spaces are small in the sense that they do not contain any Gevrey class $\mathcal{G}_{s}(\Omega)$ with $s>1$, but they are large in the sense that any initial condition $\left(u_{0}, u_{1}\right) \in V_{1}$ is the sum of two initial conditions belonging to these spaces!

Despite of the many positive results, as far as we know no paper has been devoted to negative results. In particular in the literature we found no counterexample even against the most optimistic conjecture, according to which a global solution to (1.1), (1.2), (1.3) exists assuming only that $m$ is a nonnegative continuous function, and the initial data belong to the "energy space" $V_{0}$.

In this paper we make a first step in the direction of counterexamples. We focus on local solutions, and we prove that Hirosawa's results [14] are sharp, both in the strictly hyperbolic and in the weakly hyperbolic case.

In Theorem 3.1 and Theorem 3.4 we construct indeed local solutions $u(x, t)$ of (1.1), (1.2), (1.3) with very regular initial data, but such that for every $t>0$ we have that $\left(u(x, t), u_{t}(x, t)\right)$ belongs to the phase space $V_{\beta}$ if and only if $\beta \leq 1 / 2$. In a few words these solutions exhibit an instantaneous derivative loss up to $V_{1 / 2}$. In these examples the maximal regularity admitted for the initial data depends on the continuity modulus of $m$. Just to give some examples, in the strictly hyperbolic case we have the following three situations (see Example 3.2).

- If $m$ is continuous we can have derivative loss for quasi analytic initial data. This proves in particular that in Nishihara's result [19] the Lipschitz continuity 
assumption on the nonlinear term cannot be relaxed to continuity, even when looking for local solutions.

- If $m$ is $\alpha$-Hölder continuous for some $\alpha \in(0,1)$, then we can have derivative loss for initial data in any Gevrey space $\mathcal{G}_{s}(\Omega)$ with $s>(1-\alpha)^{-1}$.

- If $m$ is $\alpha$-Hölder continuous for every $\alpha \in(0,1)$, then we can have derivative loss for initial data in $C^{\infty}(\Omega)$. This proves in particular that in the results stated in (A) (i.e., well posedness in $V_{\beta}$ for every $\beta \geq 1 / 2$ ) the Lipschitz continuity assumption on $m$ cannot be relaxed to Hölder continuity.

In the weakly hyperbolic case we have for example the following two situations (see Example 3.5).

- If $m$ is $\alpha$-Hölder continuous for some $\alpha \in(0,1)$, then we can have derivative loss for initial data in any Gevrey space $\mathcal{G}_{s}(\Omega)$ with $s>1+\alpha / 2$.

- If $m$ is Lipschitz continuous we can have derivative loss for initial data in any Gevrey space $\mathcal{G}_{s}(\Omega)$ with $s>3 / 2$. This proves in particular that in the results stated in (A) the strict hyperbolicity cannot be relaxed to weak hyperbolicity.

Our examples do not exclude that a local solution can always exist in $V_{1 / 2}$. This remains an open problem.

Our approach is based on the theory developed by F. Colombini, E. De Giorgi, and S. Spagnolo [5], and then extended by F. Colombini and S. Spagnolo [6], and by F. Colombini, E. Jannelli, and S. Spagnolo [7]. They considered linear equations with a time dependent coefficient such as

$$
u_{t t}(t, x)-c(t) \Delta u(t, x)=0 .
$$

If $c(t)$ is not Lipschitz continuous or vanishes for $t=0$, they showed how to construct "solutions" of (1.4) which are very regular at time $t=0$, but very irregular (not even distributions) for $t>0$.

The construction of our counterexamples is divided into two steps. In the first step we consider the linear equation (1.4), and we modify the parameters in the construction described in [5-7] in order to obtain solutions of (1.4) which are very regular at time $t=0$, but with a prescribed minimal regularity (they belong to $V_{\beta}$ if and only if $\beta \leq 1 / 2$ ) for every $t>0$. These counterexamples for the linear equation, stated in Proposition 3.6 and Proposition 3.7, are maybe interesting in themselves because they extend the theory developed in [5-7] to coefficients $c(t)$ with arbitrary continuity modulus. The assumptions on initial data in these counterexamples are sharp because they are complementary to those required in the existence results (both for linear and for Kirchhoff equations).

In the second step we show that these solutions, up to modifying one dominant Fourier component, are solutions of (1.1) for a suitable choice of the nonlinearity $m$.

This paper is organized as follows. In Section 2 we rephrase (1.1), (1.2), (1.3) as an abstract evolution problem in a Hilbert space, and we restate Hirosawa's local existence results in the abstract setting. In Section 3 we state our counterexamples, which we prove in Section 4. For the convenience of the reader in Appendix A we sketch the main point of the proof of the local existence results in the abstract setting. 


\section{Preliminaries and local existence results}

\section{Continuity moduli}

Let $\omega:[0,+\infty) \rightarrow[0,+\infty)$. The following assumptions are recalled throughout the paper.

$(\omega 1)$ We have that $\omega \in C^{0}([0,+\infty))$ is an increasing function such that $\omega(0)=0$, and $\omega(a+b) \leq \omega(a)+\omega(b)$ for every $a \geq 0$ and $b \geq 0$.

$(\omega 2)$ The function $\sigma \rightarrow \sigma / \omega(\sigma)$ is nondecreasing.

A function $f: X \rightarrow \mathbb{R}$ (where $X \subseteq \mathbb{R}$ ) is said to be $\omega$-continuous if there exists a constant $L \in \mathbb{R}$ such that

$$
|f(a)-f(b)| \leq L \omega(|a-b|) \quad \forall a \in X, \forall b \in X
$$

Two simple properties of continuity moduli are stated in Lemma A.2 of the appendix. In particular from (A.4) it follows that the composition of a Lipschitz continuous function and an $\omega$-continuous function (in any order) is again an $\omega$ continuous function.

It is not difficult to see that the set of $\omega$-continuous functions only depends on the values of $\omega$ in a right neighborhood of 0 . For this reason, with a little abuse of notation, we often consider continuity moduli which are defined, and satisfy $(\omega 1)$ and/or $(\omega 2)$, in a right neighborhood of 0 . For the same reason when needed we assume also that $\omega$ is invertible.

\section{Abstract setting for Kirchhoff equations}

Let $H$ be a separable real Hilbert space. For every $x$ and $y$ in $H$, let $|x|$ denote the norm of $x$, and let $\langle x, y\rangle$ denote the scalar product of $x$ and $y$. Let $A$ be an unbounded linear operator on $H$ with dense domain $D(A)$. We always assume that $A$ is self-adjoint and nonnegative, so that the power $A^{\beta}$ is defined for every $\beta \geq 0$ in a suitable domain $D\left(A^{\beta}\right)$.

We consider the second order evolution problem

$$
u^{\prime \prime}(t)+m\left(\left|A^{1 / 2} u(t)\right|^{2}\right) A u(t)=0, \quad \forall t \geq 0,
$$

with initial data

$$
u(0)=u_{0}, \quad u^{\prime}(0)=u_{1} \text {. }
$$

It is well known that (2.2), (2.3) is just an abstract setting of (1.1), (1.2), (1.3), corresponding to the case where $H=L^{2}(\Omega)$, and $A u=-\Delta u$, defined for every $u$ in a suitable domain $D(A)$ depending on the boundary conditions (see [1]). 


\section{Functional spaces}

For the sake of simplicity, let us assume that $H$ admits a countable complete orthonormal system $\left\{e_{k}\right\}_{k \geq 1}$ made by eigenvectors of $A$. We denote the corresponding eigenvalues by $\lambda_{k}^{2}$, so that $A e_{k}=\lambda_{k}^{2} e_{k}$ for every $k \geq 1$.

Under this assumption (which in the concrete case corresponds to bounded domains) we can work with Fourier series. However, any definition or statement of this section can be easily extended to the general setting just by using the spectral theorem for self-adjoint operators [22, Theorem VIII.4, page 260].

By means of the orthonormal system every $u \in H$ can be written in a unique way in the form $u=\sum_{k=1}^{\infty} u_{k} e_{k}$, where $u_{k}=\left\langle u, e_{k}\right\rangle$ are the Fourier components of $u$. Moreover for every $\beta>0$ we have that

$$
A^{\beta} u:=\sum_{k=1}^{\infty} \lambda_{k}^{2 \beta} u_{k} e_{k}
$$

so that we can consider the quantity

$$
\|u\|_{D\left(A^{\beta}\right)}^{2}:=\sum_{k=1}^{\infty} \lambda_{k}^{4 \beta} u_{k}^{2}
$$

and characterize the spaces $D\left(A^{\beta}\right)$ and $D\left(A^{\infty}\right)$ as follows

$$
D\left(A^{\beta}\right):=\left\{u \in H:\|u\|_{D\left(A^{\beta}\right)}^{2}<+\infty\right\}, \quad D\left(A^{\infty}\right):=\bigcap_{\beta>0} D\left(A^{\beta}\right) .
$$

With these notations the phase spaces $V_{\beta}$ are defined as $V_{\beta}:=D\left(A^{(\beta+1) / 2}\right) \times$ $D\left(A^{\beta / 2}\right)$.

Let now $\varphi:[0,+\infty) \rightarrow[1,+\infty)$ be any function. Then for every $r>0$ and $\beta>0$ we can set

$$
\|u\|_{\varphi, r, \beta}^{2}:=\sum_{k=1}^{\infty} \lambda_{k}^{4 \beta} u_{k}^{2} \exp \left(r \varphi\left(\lambda_{k}\right)\right)
$$

and then define the spaces

$$
\mathcal{G}_{\varphi, r, \beta}(A):=\left\{u \in H:\|u\|_{\varphi, r, \beta}^{2}<+\infty\right\}, \quad \mathcal{G}_{\varphi, \beta}(A):=\bigcup_{r>0} \mathcal{G}_{\varphi, r, \beta}(A)
$$

If two continuous functions $\varphi_{1}(\sigma)$ and $\varphi_{2}(\sigma)$ coincide for every large enough $\sigma$, then $\mathcal{G}_{\varphi_{1}, r, \beta}(A)=\mathcal{G}_{\varphi_{2}, r, \beta}(A)$. For this reason, with a little abuse of notation, we consider these spaces even if $\varphi(\sigma)$ is defined, continuous, and greater than 1 only for large values of $\sigma$. 


\section{Hirosawa's results}

We are now ready to recall the main results proved in [14], restated in the abstract setting. Since the abstract statements do not seem to follow trivially from the ones in [14], we sketch the proofs in Appendix A.

The first result concerns the strictly hyperbolic case (see [14, Theorem 2.2]).

Theorem A (Strictly hyperbolic case). Let $\omega:[0,+\infty) \rightarrow[0,+\infty)$ be a function satisfying $(\omega 1)$. Let $m:[0,+\infty) \rightarrow(0,+\infty)$ be an $\omega$-continuous function satisfying the strict hyperbolicity condition $m(\sigma) \geq v>0$ for every $\sigma \geq 0$.

Let $\varphi:[0,+\infty) \rightarrow[1,+\infty)$ be a function such that

$$
\limsup _{\sigma \rightarrow+\infty} \frac{\sigma}{\varphi(\sigma)} \omega\left(\frac{1}{\sigma}\right)<+\infty \text {. }
$$

Let us assume that

$$
\left(u_{0}, u_{1}\right) \in \mathcal{G}_{\varphi, r_{0}, 3 / 4}(A) \times \mathcal{G}_{\varphi, r_{0}, 1 / 4}(A)
$$

for some $r_{0}>0$.

Then there exist $T>0$, and $R>0$ with $R T<r_{0}$ such that problem (2.2), (2.3) admits at least one local solution

$$
u \in C^{1}\left([0, T] ; \mathcal{G}_{\varphi, r_{0}-R t, 1 / 4}(A)\right) \cap C^{0}\left([0, T] ; \mathcal{G}_{\varphi, r_{0}-R t, 3 / 4}(A)\right) .
$$

Remark 2.1. Condition (2.7), with the range space depending on time, simply means that

$$
u \in C^{1}\left([0, \tau] ; \mathcal{G}_{\varphi, r_{0}-R \tau, 1 / 4}(A)\right) \cap C^{0}\left([0, \tau] ; \mathcal{G}_{\varphi, r_{0}-R \tau, 3 / 4}(A)\right)
$$

for every $\tau \in(0, T]$. This amounts to say that scales of Hilbert spaces are the natural setting for this problem.

Example 2.2. Let us give some examples in order to clarify the interplay between assumptions (2.5) and (2.6).

- If $\omega(\sigma)=o(1)$ as $\sigma \rightarrow 0^{+}$(which simply means that $m$ is continuous), then (2.5) holds true with $\varphi(\sigma)=\sigma$. In this case (2.6) means that $u_{0}$ and $u_{1}$ are analytic, and one has re-obtained the classical local existence result for analytic data in the strictly hyperbolic case. In (2.5) one can also take $\varphi(\sigma)=\sigma \omega(1 / \sigma)$, thus obtaining local existence for a larger class of initial data.

- If $\omega(\sigma)=\sigma^{\alpha}$ for some $\alpha \in(0,1)$ (which means that $m$ is Hölder continuous), then (2.5) holds true with $\varphi(\sigma)=\sigma^{1-\alpha}$. In this case (2.6) means that one can take initial data in the Gevrey space $\mathcal{G}_{s}(A)$ with $s=(1-\alpha)^{-1}$. We recall that $\mathcal{G}_{s}(A)$ is the space $\mathcal{G}_{\varphi, \beta}(A)$ corresponding to $\varphi(\sigma)=\sigma^{1 / s}(\beta$ is insignificant in this case). 
- If $\omega(\sigma)=\sigma|\log \sigma|$ (which means that $m$ is log-Lipschitz continuous), then (2.5) holds true with $\varphi(\sigma)=\log \sigma$. In this case $\mathcal{G}_{\varphi, r, \beta}(A)=D\left(A^{\beta+r / 4}\right)$. One can therefore take initial data in $D\left(A^{\infty}\right)$ and obtain a solution in the same space, or even initial data in $V_{\gamma}$ for some $\gamma>1 / 2$ and obtain a solution with a possible progressive derivative loss (due to the term $r_{0}-R t$ in (2.7)).

- If $\omega(\sigma)=\sigma$ (which means that $m$ is Lipschitz continuous), then (2.5) holds true with $\varphi(\sigma) \equiv 1$. Therefore (2.6) means that one can take initial data in $V_{1 / 2}$. This is of course the classical existence result in Sobolev spaces.

Since we are interested in local solutions, Theorem A can be applied also in the mildly degenerate case, namely when $m$ may vanish but $m\left(\left|A^{1 / 2} u_{0}\right|^{2}\right)>0$.

The following result (see [14, Theorem 2.1]) concerns the weakly hyperbolic case, and it is essential to deal with the really degenerate case, i.e., when $m\left(\left|A^{1 / 2} u_{0}\right|^{2}\right)=0$.

Theorem B (Weakly hyperbolic case). Let $\omega:[0,+\infty) \rightarrow[0,+\infty)$ be a function satisfying $(\omega 1)$. Let $m:[0,+\infty) \rightarrow[0,+\infty)$ be an $\omega$-continuous function.

Let $\varphi:[0,+\infty) \rightarrow[1,+\infty)$ be a function such that

$$
\limsup _{\sigma \rightarrow+\infty} \sigma\left[\varphi\left(\frac{\sigma}{\sqrt{\omega(1 / \sigma)}}\right)\right]^{-1}<+\infty
$$

Let $\left(u_{0}, u_{1}\right)$ and $r_{0}>0$ be such that (2.6) holds true.

Then there exist $T>0$, and $R>0$ with $R T<r_{0}$ such that problem (2.2), (2.3) admits at least one local solution u satisfying (2.7).

Example 2.3. Let us give some examples.

- If $\omega(\sigma)=o(1)$ as $\sigma \rightarrow 0^{+}$, then (2.8) holds true with $\varphi(\sigma)=\sigma$. Once again (2.6) means that $u_{0}$ and $u_{1}$ are analytic, and one has re-obtained the classical local existence result for analytic data in the weakly hyperbolic case.

- If $\omega(\sigma)=\sigma^{\alpha}$ for some $\alpha \in(0,1]$, then $(2.8)$ holds true with $\varphi(\sigma)=\sigma^{2 /(\alpha+2)}$. Therefore (2.6) means that one can take initial data in the Gevrey space $\mathcal{G}_{S}(A)$ with $s=1+\alpha / 2$. This is true in particular for $\alpha=1$ (i.e., when $m$ is Lipschitz continuous). In this case we have local existence for initial data in $\mathcal{G}_{3 / 2}(A)$.

Remark 2.4. One cannot expect local solutions to be unique if $m$ is not Lipschitz continuous (see [12] and some examples in [2]). However the existence results rely on an a priori estimate (see Proposition A.1) which implies in particular that under assumptions (2.5) or (2.8) any local solution satisfying the minimal regularity requirement (A.1) also satisfies the stronger condition (2.7). This is a sort of propagation of regularity: if the space in (2.6) is strictly contained in $D\left(A^{3 / 4}\right) \times D\left(A^{1 / 4}\right)$, then also solutions lie in a scale of spaces which is strictly contained in $D\left(A^{3 / 4}\right) \times D\left(A^{1 / 4}\right)$. We are going to see that this is no more true when condition (2.5) or (2.8) are not satisfied. 


\section{Statements of counterexamples}

The first counterexample shows the optimality of Theorem A in the non-Lipschitz case.

Theorem 3.1 (Strictly hyperbolic case). Let $A$ be a self-adjoint linear operator on a Hilbert space $H$. Let us assume that there exist a countable (not necessarily complete) orthonormal system $\left\{e_{k}\right\}_{k \geq 1}$ in $H$, and an increasing unbounded sequence $\left\{\lambda_{k}\right\}_{k \geq 1}$ of positive real numbers such that $A e_{k}=\lambda_{k}^{2} e_{k}$ for every $k \geq 1$.

Let $\omega:[0,+\infty) \rightarrow[0,+\infty)$ be a function satisfying $(\omega 1)$ and $(\omega 2)$.

Let $\varphi:[0,+\infty) \rightarrow[1,+\infty)$ be a function such that

$$
\lim _{\sigma \rightarrow+\infty} \frac{\sigma}{\varphi(\sigma)} \omega\left(\frac{1}{\sigma}\right)=+\infty .
$$

Then there exist a function $m:[0,+\infty) \rightarrow[1 / 2,3 / 2]$, a real number $T_{0}>0$, and a function $u:\left[0, T_{0}\right] \rightarrow H$ such that

(i) $m$ is $\omega$-continuous;

(ii) $\left(u(0), u^{\prime}(0)\right) \in \mathcal{G}_{\varphi, r, 3 / 4}(A) \times \mathcal{G}_{\varphi, r, 1 / 4}(A)$ for every $r>0$;

(iii) $u \in C^{1}\left(\left[0, T_{0}\right] ; D\left(A^{1 / 4}\right)\right) \cap C^{0}\left(\left[0, T_{0}\right] ; D\left(A^{3 / 4}\right)\right)$ is a solution of $(2.2)$;

(iv) for every $t \in\left(0, T_{0}\right]$ we have that $\left(u(t), u^{\prime}(t)\right) \notin V_{\beta}$ for every $\beta>1 / 2$.

Example 3.2. Let us consider some examples.

- The assumptions of Theorem 3.1 are satisfied if we take

$$
\omega(\sigma)=\frac{1}{|\log \sigma|^{1 / 2}}, \quad \varphi(\sigma)=\frac{\sigma}{\log \sigma} .
$$

In this case $m$ is continuous, the initial data are quasi analytic, and the solution $u$ has an instantaneous infinite derivative loss.

- Let $\alpha \in(0,1)$. The assumptions of Theorem 3.1 are satisfied if we take

$$
\omega(\sigma)=\sigma^{\alpha}, \quad \varphi(\sigma)=\frac{\sigma^{1-\alpha}}{\log \sigma} .
$$

In this case $m$ is $\alpha$-Hölder continuous, the initial data are in the Gevrey space $\mathcal{G}_{s}(A)$ for every $s>(1-\alpha)^{-1}$, and the solution $u$ has an instantaneous infinite derivative loss.

- The assumptions of Theorem 3.1 are satisfied if we take

$$
\omega(\sigma)=\sigma|\log \sigma|^{3}, \quad \varphi(\sigma)=\log ^{2} \sigma .
$$

In this case $m$ is $\alpha$-Hölder continuous for every $\alpha \in(0,1)$ (but not log-Lipschitz continuous), the initial data are in $D\left(A^{\infty}\right)$, and once again the solution $u$ has an instantaneous infinite derivative loss. 
Remark 3.3. Theorem 3.1, as it is stated, is void in the log-Lipschitz case. Indeed when $\omega(\sigma)=\sigma|\log \sigma|$ and $\varphi$ satisfies (3.1), then all initial data satisfying (ii) belong to $V_{1 / 2}$ but not to $V_{\beta}$ for $\beta>1 / 2$. In a certain sense they have no derivatives to loose!

On the other hand, a careful inspection of the proof reveals that Theorem 3.1 can be improved as follows. Given any function $\psi:[0,+\infty) \rightarrow[1,+\infty)$ such that $\psi(\sigma) \rightarrow+\infty$ as $\sigma \rightarrow+\infty$ we can find a solution satisfying (i), (ii), (iii), and

$\left(\mathrm{iv}^{\prime}\right)$ for every $t \in\left(0, T_{0}\right]$ we have that $\left(u(t), u^{\prime}(t)\right) \notin \mathcal{G}_{\psi, r, 3 / 4}(A) \times \mathcal{G}_{\psi, r, 1 / 4}(A)$ for every $r>0$.

Thus for example we can take

$$
\omega(\sigma)=\sigma|\log \sigma|, \quad \varphi(\sigma)=\log |\log \sigma|, \quad \psi(\sigma)=\log |\log | \log \sigma||,
$$

and obtain a solution with initial data in $\mathcal{G}_{\varphi, r, 3 / 4}(A) \times \mathcal{G}_{\varphi, r, 1 / 4}(A)$ which for every positive time does not belong even to the weaker scale $\mathcal{G}_{\psi, r, 3 / 4}(A) \times \mathcal{G}_{\psi, r, 1 / 4}(A)$. So also in the log-Lipschitz case we have a (infinitesimally small) derivative loss.

We spare the reader from this generalization.

The second counterexample shows the optimality of Theorem B.

Theorem 3.4 (Weakly hyperbolic case). Let $A,\left\{e_{k}\right\},\left\{\lambda_{k}\right\}$ be as in Theorem 3.1.

Let $\omega:[0,+\infty) \rightarrow[0,+\infty)$ be a function satisfying $(\omega 1)$ and $(\omega 2)$.

Let $\varphi:[0,+\infty) \rightarrow[1,+\infty)$ be a function such that

$$
\lim _{\sigma \rightarrow+\infty} \sigma\left[\varphi\left(\frac{\sigma}{\sqrt{\omega(1 / \sigma)}}\right)\right]^{-1}=+\infty .
$$

Then there exist a function $m:[0,+\infty) \rightarrow[0,3 / 2]$, a real number $T_{0}>0$, and a function $u:\left[0, T_{0}\right] \rightarrow H$ such that

(i) $m$ is $\omega$-continuous;

(ii) $\left(u(0), u^{\prime}(0)\right) \in \mathcal{G}_{\varphi, r, 3 / 4}(A) \times \mathcal{G}_{\varphi, r, 1 / 4}(A)$ for every $r>0$;

(iii) $u \in C^{1}\left(\left[0, T_{0}\right] ; D\left(A^{1 / 4}\right)\right) \cap C^{0}\left(\left[0, T_{0}\right] ; D\left(A^{3 / 4}\right)\right)$ is a solution of (2.2);

(iv) for every $t \in\left(0, T_{0}\right]$ we have that $\left(u(t), u^{\prime}(t)\right) \notin V_{\beta}$ for every $\beta \geq 1$;

(v) there exists a sequence $\tau_{k} \rightarrow 0^{+}$such that $\left|A^{(\beta+1) / 2} u\left(\tau_{k}\right)\right|$ is unbounded for every $\beta>1 / 2$.

Example 3.5. Let $\alpha \in(0,1]$. The assumptions of Theorem 3.1 are satisfied if we take

$$
\omega(\sigma)=\sigma^{\alpha}, \quad \varphi(\sigma)=\frac{\sigma^{2 /(\alpha+2)}}{\log \sigma} .
$$

In this case $m$ is $\alpha$-Hölder continuous (Lipschitz continuous if $\alpha=1$ ), the initial data are in the Gevrey space $\mathcal{G}_{s}(A)$ for every $s>1+\alpha / 2$, and the solution $u$ has an instantaneous infinite derivative loss.

In particular, in contrast to the strictly hyperbolic case (see Remark 3.3), Theorem 3.4 provides examples of infinite derivative loss even in the Lipschitz (or log-Lipschitz) case. 
The counterexamples of Theorem 3.1 and Theorem 3.4 originate from two counterexamples for the linear equation

$$
v^{\prime \prime}+c(t) A v=0 .
$$

This equation is well studied in mathematical literature. It is well known for example that, if the coefficient $c(t)$ is $\omega$-continuous, then the Cauchy problem is well posed in $\mathcal{G}_{\varphi, \beta}(A)$ provided that $\varphi$ and $\omega$ satisfy (2.5) in the strictly hyperbolic case, and (2.8) in the weakly hyperbolic case. The main argument is that the approximated energy estimates introduced in [5] can be extended word for word to arbitrary continuity moduli as we do in the appendix below.

This result is sharp. Indeed if $\varphi$ and $\omega$ do not satisfy (2.5) or (2.8), hence if they satisfy (3.1) or (3.2) (see also Remark 3.9 below), then the Cauchy problem is not well posed in $\mathcal{G}_{\varphi, \beta}(A)$. In literature we found a lot of counterexamples for special choices of $\omega$ and $\varphi$ (see for example [4] and the references quoted therein), but we didn't find the general counterexamples under assumptions (3.1) or (3.2).

In the following two propositions we state them in the form which is needed in the proof of Theorem 3.1 and Theorem 3.4.

Proposition 3.6 (Strictly hyperbolic case). Let $A,\left\{e_{k}\right\},\left\{\lambda_{k}\right\}, \omega, \varphi$ be as in Theorem 3.1.

Then there exist $c:[0,1] \rightarrow[1 / 2,3 / 2]$, and $v:[0,1] \rightarrow H$ such that

(SH-1) $c$ is $\omega$-continuous;

$(\mathrm{SH}-2)\left(v(0), v^{\prime}(0)\right) \in \mathcal{G}_{\varphi, r, 3 / 4}(A) \times \mathcal{G}_{\varphi, r, 1 / 4}(A)$ for every $r>0$;

(SH-3) $v \in C^{1}\left([0,1] ; D\left(A^{1 / 4}\right)\right) \cap C^{0}\left([0,1] ; D\left(A^{3 / 4}\right)\right)$ is a solution of $(3.3)$;

(SH-4) for every $t \in(0,1]$ we have that $\left(v(t), v^{\prime}(t)\right) \notin V_{\beta}$ for every $\beta>1 / 2$.

Proposition 3.7 (Weakly hyperbolic case). Let $A,\left\{e_{k}\right\},\left\{\lambda_{k}\right\}, \omega, \varphi$ be as in Theorem 3.4.

Then there exist $c:[0,1] \rightarrow[0,3 / 2]$, and $v:[0,1] \rightarrow H$ such that

(WH-1) c is $\omega$-continuous;

$(\mathrm{WH}-2)\left(v(0), v^{\prime}(0)\right) \in \mathcal{G}_{\varphi, r, 3 / 4}(A) \times \mathcal{G}_{\varphi, r, 1 / 4}(A)$ for every $r>0$;

(WH-3) $v \in C^{1}\left([0,1] ; D\left(A^{1 / 4}\right)\right) \cap C^{0}\left([0,1] ; D\left(A^{3 / 4}\right)\right)$ is a solution of $(3.3)$;

(WH-4) for every $t \in(0,1]$ we have that $\left(v(t), v^{\prime}(t)\right) \notin V_{\beta}$ for every $\beta \geq 1$;

(WH-5) there exists a sequence $\tau_{k} \rightarrow 0^{+}$such that $\left|A^{(\beta+1) / 2} v\left(\tau_{k}\right)\right|$ is unbounded for every $\beta>1 / 2$.

Remark 3.8. Proposition 3.6 and Proposition 3.7 are strongly based on the counterexamples shown in [5-7]. On the other hand, besides the fact that we deal with arbitrary continuity moduli, and arbitrary sequences of eigenvalues, there are some differences we would like to point out.

- In [5-7] the derivative loss is bigger because solutions instantaneously lie outside the space of distributions. Here we need to be more careful since we want solutions to lie in $V_{1 / 2}$ and nothing more. 
- "Derivative loss" has a slightly different meaning in [5-7] and in this paper. Losing the $m$-th derivative in [5-7] means that there exists a sequence $\tau_{k} \rightarrow 0^{+}$ such that the norm of $\left(v\left(\tau_{k}\right), v^{\prime}\left(\tau_{k}\right)\right)$ in $V_{m}$ tends to $+\infty$. This of course may happen also if $v\left(\tau_{k}\right)$ and $v^{\prime}\left(\tau_{k}\right)$ are in $D\left(A^{\infty}\right)$ for every $k$. In other words, what is actually lost is a uniform bound on the $m$-th derivative.

In statements (SH-4) and (WH-4), and in the corresponding statements of Theorem 3.1 and Theorem 3.4, we lose the $m$-th derivative in a stronger sense, namely $\left(v(t), v^{\prime}(t)\right) \notin V_{m}$ for every $t>0$. On the contrary in statement (WH-5), and in the corresponding statement of Theorem 3.4, we are forced to lose the last derivatives only in the weaker sense.

Remark 3.9. A careful inspection of the proofs shows that the same conclusions hold true also if the limit in (3.1) and (3.2) is replaced by the corresponding limsup computed along the sequence $\left\{\lambda_{k}\right\}$.

\section{Proofs}

The proof is organized as follows. In Section 4.1 we construct functions $c(t)$ and $v(t)$ depending on several parameters. In Proposition 4.2, Proposition 4.3 and Corollary 4.4 we relate the regularity properties of $c(t)$ and $v(t)$ to suitable conditions on the parameters. Then in Section 4.2 we choose the parameters in order to prove Proposition 3.6. In Section 4.3 we do the same for Proposition 3.7. Finally in Section 4.4 we prove Theorem 3.1 and Theorem 3.4.

\subsection{General Construction}

\section{Ingredients}

The starting point of the construction are the functions

$$
\begin{aligned}
b(\varepsilon, t) & :=1-4 \varepsilon \sin (2 t)-\varepsilon^{2}(1-\cos (2 t))^{2}, \\
w(\varepsilon, t) & :=\sin t \cdot \exp \left(\varepsilon\left(t-\frac{1}{2} \sin (2 t)\right)\right),
\end{aligned}
$$

introduced in Section 7 of [5].

Then the construction is based on seven sequences $\left\{t_{k}\right\},\left\{s_{k}\right\},\left\{\tau_{k}\right\},\left\{\eta_{k}\right\},\left\{\delta_{k}\right\}$, $\left\{\varepsilon_{k}\right\},\left\{a_{k}\right\}$ satisfying the following assumptions.

$\left(\mathrm{Hp}-t_{k}\right)$ The sequence $\left\{t_{k}\right\}$ is a decreasing sequence of positive real numbers (which we think as times) such that $t_{0}=1$, and $t_{k} \rightarrow 0^{+}$as $k \rightarrow+\infty$.

$\left(\mathrm{Hp}-s_{k}\right)$ The sequence $\left\{s_{k}\right\}$ is a sequence of positive real numbers (which we think as times) such that $t_{k}<s_{k}<t_{k-1}$ for every $k \geq 1$.

$\left(\mathrm{Hp}-\tau_{k}\right)$ The sequence $\left\{\tau_{k}\right\}$ is a sequence of positive real numbers (which we think as times) such that $t_{k}<\tau_{k}<s_{k}$ for every $k \geq 1$.

$\left(\mathrm{Hp}-\eta_{k}\right)$ The sequence $\left\{\eta_{k}\right\}$ is an increasing subsequence of the sequence $\left\{\lambda_{k}\right\}$ of the eigenvalues of $A^{1 / 2}$. 
(Hp- $\left.\delta_{k}\right)$ The sequence $\left\{\delta_{k}\right\}$ is a nonincreasing sequence of positive real numbers with $\delta_{0}=1$. Moreover we require that $\sqrt{\delta_{k}} \eta_{k} t_{k} /(2 \pi)$ and $\sqrt{\delta_{k}} \eta_{k} s_{k} /(2 \pi)$ are integers, and $2 \sqrt{\delta_{k}} \eta_{k} \tau_{k} / \pi$ is an odd integer for every $k \geq 1$.

(Hp- $\varepsilon_{k}$ ) The sequence $\left\{\varepsilon_{k}\right\}$ is a sequence of positive real numbers such that $\varepsilon_{k} \leq 1 / 16$ for every $k \geq 1$. Moreover we require that $\varepsilon_{k} \delta_{k} \rightarrow 0$ and that $\left\{\sqrt{\delta_{k}} \varepsilon_{k} \eta_{k}\right\}$ is a nondecreasing sequence.

No special assumption is required on the sequence $\left\{a_{k}\right\}$. We denote the limit of $\left\{\delta_{k}\right\}$ by $\delta_{\infty}$.

\section{Definition and properties of $c(t)$}

Let $c:[0,1] \rightarrow \mathbb{R}$ be the function defined by $c(0)=\delta_{\infty}$, and for every $k \geq 1$

$$
c(t):= \begin{cases}\delta_{k} b\left(\varepsilon_{k}, \sqrt{\delta_{k}} \eta_{k} t\right) & \text { if } t \in\left[t_{k}, s_{k}\right], \\ \frac{\delta_{k-1}-\delta_{k}}{t_{k-1}-s_{k}}\left(t-s_{k}\right)+\delta_{k} & \text { if } t \in\left[s_{k}, t_{k-1}\right] .\end{cases}
$$

Note that in the interval $\left[s_{k}, t_{k-1}\right]$ the function $c(t)$ is the affine interpolation between $\delta_{k}$ and $\delta_{k-1}$.

From the assumptions on the parameters we have that $c\left(t_{k}\right)=c\left(s_{k}\right)=\delta_{k}$. Moreover

$$
\begin{aligned}
\delta_{k}-8 \varepsilon_{k} \delta_{k} & \leq c(t) \leq \delta_{k}+8 \varepsilon_{k} \delta_{k} & & \forall t \in\left[t_{k}, s_{k}\right], \\
\delta_{k} & \leq c(t) \leq \delta_{k-1} & & \forall t \in\left[s_{k}, t_{k-1}\right] .
\end{aligned}
$$

Since $\varepsilon_{k} \delta_{k} \rightarrow 0$, and $\delta_{k} \rightarrow \delta_{\infty}$, estimates (4.4) and (4.5) imply that $c(t)$ is continuous in $[0,1]$. Since $\varepsilon_{k} \leq 1 / 16$ we have also that

$$
\frac{1}{2} \delta_{k} \leq c(t) \leq \frac{3}{2} \delta_{k} \quad \forall t \in\left[t_{k}, s_{k}\right],
$$

and globally

$$
\frac{1}{2} \delta_{\infty} \leq c(t) \leq \frac{3}{2} \quad \forall t \in[0,1] .
$$

Concerning the derivative we have that

$$
\left|c^{\prime}(t)\right| \leq 16 \varepsilon_{k} \eta_{k} \delta_{k}^{3 / 2} \quad \forall t \in\left(t_{k}, s_{k}\right),
$$

and of course

$$
c^{\prime}(t)=\frac{\delta_{k-1}-\delta_{k}}{t_{k-1}-s_{k}} \quad \forall t \in\left(s_{k}, t_{k-1}\right) .
$$

The $\omega$-continuity of $c(t)$ in the whole interval $[0,1]$ can be deduced from the $\omega$ continuity in the intervals $\left[t_{k}, t_{k-1}\right]$ provided that the following uniform estimates hold true (we omit the easy and classical proof).

Lemma 4.1. Let $c:[0,1] \rightarrow \mathbb{R}$ be any function. Let $\left\{t_{k}\right\}$ be any sequence satisfying $\left(\mathrm{Hp}-t_{k}\right)$. 
Then $c$ is $\omega$-continuous in $[0,1]$ if and only if there exists a constant $L$ such that

(i) $\left|c\left(t_{i}\right)-c\left(t_{j}\right)\right| \leq L \omega\left(\left|t_{i}-t_{j}\right|\right)$ for every pair of positive integers $i$ and $j$;

(ii) $|c(a)-c(b)| \leq L \omega(|a-b|)$ for every $k \geq 1$, and every $a$ and $b$ in $\left[t_{k}, t_{k-1}\right]$.

Applying the lemma we find the following sufficient condition for the for the $\omega$ continuity of $c(t)$.

Proposition 4.2. The function $c(t)$ defined in (4.3) is $\omega$-continuous in $[0,1]$ if

$$
\sup _{i<j} \frac{\delta_{i}-\delta_{j}}{\omega\left(t_{i}-t_{j}\right)}+\sup _{k \geq 1} \frac{\delta_{k-1}-\delta_{k}}{\omega\left(t_{k-1}-s_{k}\right)}+\sup _{k \geq 1} \frac{\varepsilon_{k} \delta_{k}}{\omega\left(2 \pi /\left(\eta_{k} \sqrt{\delta_{k}}\right)\right)}<+\infty .
$$

Proof. We apply Lemma 4.1. Assumption (i) is satisfied because the first supremum in (4.10) is finite. In order to verify assumption (ii) we consider separately the subintervals $\left[t_{k}, s_{k}\right]$ and $\left[s_{k}, t_{k-1}\right]$. Thanks to $(\omega 2)$ in $\left[s_{k}, t_{k-1}\right]$ we have that

$$
\frac{|c(a)-c(b)|}{\omega(|a-b|)}=\frac{\delta_{k-1}-\delta_{k}}{t_{k-1}-s_{k}} \cdot \frac{|a-b|}{\omega(|a-b|)} \leq \frac{\delta_{k-1}-\delta_{k}}{\omega\left(t_{k-1}-s_{k}\right)} .
$$

This is bounded uniformly in $k$ because the second supremum in (4.10) is finite.

In $\left[t_{k}, s_{k}\right]$ the function $c(t)$ is periodic, hence we can limit ourselves to consider points $a$ and $b$ with $|a-b|$ less or equal than a period $2 \pi /\left(\eta_{k} \sqrt{\delta_{k}}\right)$. By (4.8) and $(\omega 2)$ we therefore have that

$$
\begin{aligned}
\frac{|c(a)-c(b)|}{\omega(|a-b|)} & =\left|\frac{c(a)-c(b)}{a-b}\right| \cdot \frac{|a-b|}{\omega(|a-b|)} \\
& \leq 16 \varepsilon_{k} \eta_{k} \delta_{k}^{3 / 2} \cdot \frac{2 \pi}{\eta_{k} \sqrt{\delta_{k}}} \cdot \frac{1}{\omega\left(2 \pi /\left(\eta_{k} \sqrt{\delta_{k}}\right)\right)}
\end{aligned}
$$

This is bounded uniformly in $k$ because the third supremum in (4.10) is finite.

\section{Definition and properties of $v_{k}(t)$}

Let $v_{k}:[0,1] \rightarrow \mathbb{R}$ be the solution of the linear ordinary differential equation

$$
v_{k}^{\prime \prime}(t)+\eta_{k}^{2} c(t) v_{k}(t)=0
$$

with "initial” data

$$
v_{k}\left(t_{k}\right)=0, \quad v_{k}^{\prime}\left(t_{k}\right)=\eta_{k} \sqrt{\delta_{k}} .
$$

In order to estimate $v_{k}$ we consider the usual "Kovalevskian" energy

$$
E_{k}(t):=\left|v_{k}^{\prime}(t)\right|^{2}+\eta_{k}^{2}\left|v_{k}(t)\right|^{2},
$$


and the usual hyperbolic energy

$$
F_{k}(t):=\left|v_{k}^{\prime}(t)\right|^{2}+\eta_{k}^{2} c(t)\left|v_{k}(t)\right|^{2} .
$$

It is simple to show that their time derivatives are given by

$$
\begin{aligned}
E_{k}^{\prime}(t) & =2 \eta_{k}^{2}(1-c(t)) v_{k}(t) v_{k}^{\prime}(t) ; \\
F_{k}^{\prime}(t) & =\eta_{k}^{2} c^{\prime}(t)\left|v_{k}(t)\right|^{2} .
\end{aligned}
$$

Now we estimate $E_{k}(t)$ in four cases.

Case 1: $t \in\left[t_{k}, s_{k}\right]$

In this interval we have the explicit expression

$$
v_{k}(t)=\exp \left(-\varepsilon_{k} \eta_{k} \sqrt{\delta_{k}} t_{k}\right) \cdot w\left(\varepsilon_{k}, \eta_{k} \sqrt{\delta_{k}} t\right),
$$

which is indeed the main motivation of definitions (4.1) and (4.2). In particular we have the following equalities

$$
\begin{gathered}
E_{k}\left(t_{k}\right)=F_{k}\left(t_{k}\right)=\delta_{k} \eta_{k}^{2}, \\
E_{k}\left(s_{k}\right)=F_{k}\left(s_{k}\right)=\delta_{k} \eta_{k}^{2} \exp \left(2 \varepsilon_{k} \eta_{k} \sqrt{\delta_{k}}\left(s_{k}-t_{k}\right)\right),
\end{gathered}
$$

and the estimate

$$
E_{k}(t) \leq 3 \eta_{k}^{2} \exp \left(2 \varepsilon_{k} \eta_{k} \sqrt{\delta_{k}}\left(s_{k}-t_{k}\right)\right) \quad \forall t \in\left[t_{k}, s_{k}\right]
$$

From the explicit expression, and our assumption that $2 \sqrt{\delta_{k}} \eta_{k} \tau_{k} / \pi$ is an odd integer, we have also that

$$
\left|v_{k}\left(\tau_{k}\right)\right|=\exp \left(\varepsilon_{k} \eta_{k} \sqrt{\delta_{k}}\left(\tau_{k}-t_{k}\right)\right)
$$

Case 2: $t \in\left[s_{k}, t_{k-1}\right]$

In this interval $c^{\prime}(t) \geq 0$, and therefore from (4.16) we have that

$$
F_{k}^{\prime}(t) \leq \frac{c^{\prime}(t)}{c(t)} F_{k}(t) \quad \forall t \in\left[s_{k}, t_{k-1}\right] .
$$

Integrating in $\left[s_{k}, t\right]$ we find that

$$
\begin{aligned}
F_{k}(t) \leq F_{k}\left(s_{k}\right) \exp \left(\int_{s_{k}}^{t} \frac{c^{\prime}(s)}{c(s)} d s\right) & =F_{k}\left(s_{k}\right) \exp \left(\log \frac{c(t)}{c\left(s_{k}\right)}\right) \\
& =F_{k}\left(s_{k}\right) \cdot \frac{c(t)}{\delta_{k}}
\end{aligned}
$$


for every $t \in\left[s_{k}, t_{k-1}\right]$. Using (4.18), and the fact that $c(t) \leq \delta_{k-1}$ in this interval, we obtain in particular that

$$
F_{k}(t) \leq \delta_{k-1} \eta_{k}^{2} \exp \left(2 \varepsilon_{k} \eta_{k} \sqrt{\delta_{k}}\left(s_{k}-t_{k}\right)\right) \quad \forall t \in\left[s_{k}, t_{k-1}\right] .
$$

On the other hand we have that $F_{k}^{\prime}(t) \geq 0$ in this interval, hence

$$
F_{k}(t) \geq F_{k}\left(s_{k}\right)=\delta_{k} \eta_{k}^{2} \exp \left(2 \varepsilon_{k} \eta_{k} \sqrt{\delta_{k}}\left(s_{k}-t_{k}\right)\right) \quad \forall t \in\left[s_{k}, t_{k-1}\right]
$$

Since $c(t) \leq 3 / 2$ we have also that

$$
E_{k}(t) \leq \frac{3}{2} \cdot \frac{1}{c(t)} F_{k}(t) \quad \forall t \in(0,1],
$$

which combined with (4.21) and (4.18) gives

$$
E_{k}(t) \leq \frac{3}{2} \eta_{k}^{2} \exp \left(2 \varepsilon_{k} \eta_{k} \sqrt{\delta_{k}}\left(s_{k}-t_{k}\right)\right) \quad \forall t \in\left[s_{k}, t_{k-1}\right]
$$

Case 3: $t \in\left[0, t_{k}\right]$

From (4.15) and (4.7) we have that

$$
\left|E_{k}^{\prime}(t)\right| \leq \eta_{k}|1-c(t)| E_{k}(t) \leq \eta_{k} E_{k}(t) \quad \forall t \in[0,1] .
$$

Integrating in $\left[t, t_{k}\right]$ we obtain that

$$
E_{k}\left(t_{k}\right) \exp \left(-\eta_{k}\left(t_{k}-t\right)\right) \leq E_{k}(t) \leq E_{k}\left(t_{k}\right) \exp \left(\eta_{k}\left(t_{k}-t\right)\right) \quad \forall t \in\left[0, t_{k}\right],
$$

hence by (4.17) we conclude that

$$
\delta_{k} \eta_{k}^{2} \exp \left(-\eta_{k} t_{k}\right) \leq E_{k}(t) \leq \delta_{k} \eta_{k}^{2} \exp \left(\eta_{k} t_{k}\right) \quad \forall t \in\left[0, t_{k}\right]
$$

Case 4: $t \in\left[t_{k-1}, 1\right]$

From (4.16) we have that

$$
F_{k}^{\prime}(t)=\frac{c^{\prime}(t)}{c(t)} \cdot \eta_{k}^{2} c(t)\left|v_{k}(t)\right|^{2} \leq \frac{\left|c^{\prime}(t)\right|}{c(t)} F_{k}(t)
$$

hence

$$
F_{k}(t) \leq F_{k}\left(t_{k-1}\right) \exp \left(\int_{t_{k-1}}^{1} \frac{\left|c^{\prime}(s)\right|}{c(s)} d s\right) \quad \forall t \in\left[t_{k-1}, 1\right]
$$


Now we observe that

$$
\int_{t_{k-1}}^{1} \frac{\left|c^{\prime}(s)\right|}{c(s)} d s=\sum_{i=1}^{k-1} \int_{t_{i}}^{s_{i}} \frac{\left|c^{\prime}(s)\right|}{c(s)} d s+\sum_{i=1}^{k-1} \int_{s_{i}}^{t_{i-1}} \frac{\left|c^{\prime}(s)\right|}{c(s)} d s,
$$

and we estimate the two summands in the right hand side. From (4.8) and (4.6) we have that

$$
\frac{\left|c^{\prime}(s)\right|}{c(s)} \leq \frac{16 \varepsilon_{i} \eta_{i} \delta_{i}^{3 / 2}}{\delta_{i} / 2}=32 \varepsilon_{i} \eta_{i} \sqrt{\delta_{i}} \quad \forall s \in\left[t_{i}, s_{i}\right]
$$

By $\left(\mathrm{Hp}-\varepsilon_{k}\right)$ the sequence $\varepsilon_{k} \eta_{k} \sqrt{\delta_{k}}$ is nondecreasing. Therefore we have that

$$
\sum_{i=1}^{k-1} \int_{t_{i}}^{s_{i}} \frac{\left|c^{\prime}(s)\right|}{c(s)} d s \leq 32 \varepsilon_{k-1} \eta_{k-1} \sqrt{\delta_{k-1}} \sum_{i=1}^{k-1}\left|s_{i}-t_{i}\right| \leq 32 \varepsilon_{k-1} \eta_{k-1} \sqrt{\delta_{k-1}} .
$$

On the other hand, in the interval $\left[s_{i}, t_{i-1}\right]$ we have that $c^{\prime}(s)>0$, hence

$$
\int_{s_{i}}^{t_{i-1}} \frac{\left|c^{\prime}(s)\right|}{c(s)} d s=\int_{s_{i}}^{t_{i-1}} \frac{c^{\prime}(s)}{c(s)} d s=\log \frac{c\left(t_{i-1}\right)}{c\left(s_{i}\right)}=\log \frac{\delta_{i-1}}{\delta_{i}}
$$

and therefore (we recall that $\delta_{0}=1$ )

$$
\sum_{i=1}^{k-1} \int_{s_{i}}^{t_{i-1}} \frac{\left|c^{\prime}(s)\right|}{c(s)} d s=\sum_{i=1}^{k-1} \log \frac{\delta_{i-1}}{\delta_{i}}=\log \left(\prod_{i=1}^{k-1} \frac{\delta_{i-1}}{\delta_{i}}\right)=\log \frac{1}{\delta_{k-1}}
$$

Coming back to (4.27) and (4.26) we have proved that

$$
F_{k}(t) \leq F_{k}\left(t_{k-1}\right) \frac{1}{\delta_{k-1}} \exp \left(32 \varepsilon_{k-1} \eta_{k-1} \sqrt{\delta_{k-1}}\right) .
$$

Taking (4.22) into account we finally obtain that

$$
F_{k}(t) \leq \eta_{k}^{2} \exp \left(2 \varepsilon_{k} \eta_{k} \sqrt{\delta_{k}}\left(s_{k}-t_{k}\right)+32 \varepsilon_{k-1} \eta_{k-1} \sqrt{\delta_{k-1}}\right) \quad \forall t \in\left[t_{k-1}, 1\right] .
$$

In an analogous way we can prove that

$$
\begin{aligned}
F_{k}(t) & \geq F_{k}\left(t_{k-1}\right) \exp \left(-\int_{t_{k-1}}^{1} \frac{\left|c^{\prime}(s)\right|}{c(s)} d s\right) \\
& \geq F_{k}\left(t_{k-1}\right) \delta_{k-1} \exp \left(-32 \varepsilon_{k-1} \eta_{k-1} \sqrt{\delta_{k-1}}\right)
\end{aligned}
$$

which by (4.23) gives

$$
F_{k}(t) \geq \delta_{k} \delta_{k-1} \eta_{k}^{2} \exp \left(2 \varepsilon_{k} \eta_{k} \sqrt{\delta_{k}}\left(s_{k}-t_{k}\right)-32 \varepsilon_{k-1} \eta_{k-1} \sqrt{\delta_{k-1}}\right) \quad \forall t \in\left[t_{k-1}, 1\right] .
$$


In order to obtain estimates on $E_{k}(t)$ we just remark that

$$
\frac{\delta_{k-1}}{2} \leq c(t) \leq \frac{3}{2} \quad \forall t \in\left[t_{k-1}, 1\right]
$$

(here we used that the sequence $\delta_{k}$ is nonincreasing), so that

$$
\frac{2}{3} F_{k}(t) \leq E_{k}(t) \leq \frac{3}{\delta_{k-1}} F_{k}(t) .
$$

Therefore (4.28) and (4.29) imply the following estimates for every $t \in\left[t_{k-1}, 1\right]$

$$
\begin{aligned}
& E_{k}(t) \leq \frac{3 \eta_{k}^{2}}{\delta_{k-1}} \exp \left(2 \varepsilon_{k} \eta_{k} \sqrt{\delta_{k}}\left(s_{k}-t_{k}\right)+32 \varepsilon_{k-1} \eta_{k-1} \sqrt{\delta_{k-1}}\right) \\
& E_{k}(t) \geq \frac{2}{3} \delta_{k} \delta_{k-1} \eta_{k}^{2} \exp \left(2 \varepsilon_{k} \eta_{k} \sqrt{\delta_{k}}\left(s_{k}-t_{k}\right)-32 \varepsilon_{k-1} \eta_{k-1} \sqrt{\delta_{k-1}}\right)
\end{aligned}
$$

\section{Definition and properties of $v(t)$}

Let $e_{i_{k}}$ denote the eigenvector of $A$ corresponding to the eigenvalue $\eta_{k}^{2}$. We set

$$
v(t):=\sum_{k=1}^{\infty} a_{k} v_{k}(t) e_{i_{k}}
$$

At this level of generality this definition is purely formal, because we have no information about the convergence of the series. All regularity properties of $v(t)$ are related to convergence properties of suitable series. In particular for every $t \in[0,1]$, and every $\beta \geq 0, \varphi:[0,+\infty) \rightarrow[1,+\infty)$, and $r>0$ we have that

$$
\begin{gathered}
\left(v(t), v^{\prime}(t)\right) \in V_{\beta} \Longleftrightarrow \sum_{k=1}^{\infty} a_{k}^{2} \eta_{k}^{2 \beta} E_{k}(t)<+\infty, \\
\left(v(t), v^{\prime}(t)\right) \in \mathcal{G}_{\varphi, r, 3 / 4}(A) \times \mathcal{G}_{\varphi, r, 1 / 4}(A) \Longleftrightarrow \sum_{k=1}^{\infty} a_{k}^{2} \eta_{k} E_{k}(t) \exp \left(r \varphi\left(\eta_{k}\right)\right)<+\infty(4.34)
\end{gathered}
$$

Combining these implications with our estimates on $E_{k}(t)$ we obtain sufficient conditions in terms of the parameters for the regularity or non regularity of $v(t)$.

Proposition 4.3. Let $v(t)$ be the function defined in (4.32).

(1) For every $r>0$ we have that $\left(v(0), v^{\prime}(0)\right) \in \mathcal{G}_{\varphi, r, 3 / 4}(A) \times \mathcal{G}_{\varphi, r, 1 / 4}(A)$ if

$$
\sum_{k=1}^{\infty} a_{k}^{2} \delta_{k} \eta_{k}^{3} \exp \left(\eta_{k} t_{k}+r \varphi\left(\eta_{k}\right)\right)<+\infty
$$


(2) For every $t>0$ and $\beta \geq 0$ we have that $\left(v(t), v^{\prime}(t)\right) \in V_{\beta}$ if

$$
\sum_{k=1}^{\infty} \frac{a_{k}^{2} \eta_{k}^{2 \beta+2}}{\delta_{k-1}} \exp \left(2 \varepsilon_{k} \eta_{k} \sqrt{\delta_{k}}\left(s_{k}-t_{k}\right)+32 \varepsilon_{k-1} \eta_{k-1} \sqrt{\delta_{k-1}}\right)<+\infty
$$

(3) For every $t>0$ and $\beta \geq 0$ we have that $\left(v(t), v^{\prime}(t)\right) \notin V_{\beta}$ if

$$
\sum_{k=1}^{\infty} a_{k}^{2} \delta_{k} \delta_{k-1} \eta_{k}^{2 \beta+2} \exp \left(2 \varepsilon_{k} \eta_{k} \sqrt{\delta_{k}}\left(s_{k}-t_{k}\right)-32 \varepsilon_{k-1} \eta_{k-1} \sqrt{\delta_{k-1}}\right)=+\infty
$$

(4) We have that $v \in C^{1}\left([0,1] ; D\left(A^{1 / 4}\right)\right) \cap C^{0}\left([0,1] ; D\left(A^{3 / 4}\right)\right)$ if (4.35) holds true with $r=0$, and (4.36) holds true with $\beta=1 / 2$.

(5) For every $\beta \geq 0$ we have that the sequence $\left|A^{(\beta+1) / 2} v\left(\tau_{k}\right)\right|$ is unbounded if

$$
\lim _{k \rightarrow+\infty} a_{k}^{2} \eta_{k}^{2 \beta+2} \exp \left(2 \varepsilon_{k} \eta_{k} \sqrt{\delta_{k}}\left(\tau_{k}-t_{k}\right)\right)=+\infty
$$

Proof. Statement (1) follows combining (4.34) and (4.25) with $t=0$.

Let us examine statements (2) and (3). For $t \in(0,1]$ we have that $t \geq t_{k-1}$ for every large enough $k$, and therefore the relevant estimates are (4.30) and (4.31). At this point statement (2) follows combining (4.33) with (4.30), statement (3) follows by combining (4.33) with (4.31).

Let us consider now statement (4). A sufficient condition in order to prove that $v(t)$ has the required time regularity is that

$$
\sum_{k=1}^{\infty} a_{k}^{2} \eta_{k} \sup _{t \in[0,1]} E_{k}(t)<+\infty
$$

Now for every $k \geq 1$ we have that

$$
\sup _{t \in[0,1]} E_{k}(t) \leq \delta_{k} \eta_{k}^{2} \exp \left(\eta_{k} t_{k}\right)+\frac{3 \eta_{k}^{2}}{\delta_{k-1}} \exp \left(2 \varepsilon_{k} \eta_{k} \sqrt{\delta_{k}}\left(s_{k}-t_{k}\right)+32 \varepsilon_{k-1} \eta_{k-1} \sqrt{\delta_{k-1}}\right) .
$$

This estimate indeed easily follows from (4.25) if $t \leq t_{k}$, from (4.19) or (4.24) if $t_{k} \leq t \leq t_{k-1}$, from (4.30) if $t_{k-1} \leq t \leq 1$.

Therefore (4.39) follows from (4.35) with $r=0$ and (4.36) with $\beta=1 / 2$.

Let us finally prove statement (5). By (4.20) we have that

$$
\left|A^{(\beta+1) / 2} v\left(\tau_{k}\right)\right|^{2} \geq a_{k}^{2} \eta_{k}^{2 \beta+2}\left|v_{k}\left(\tau_{k}\right)\right|^{2}=a_{k}^{2} \eta_{k}^{2 \beta+2} \exp \left(2 \varepsilon_{k} \eta_{k} \sqrt{\delta_{k}}\left(\tau_{k}-t_{k}\right)\right),
$$

so that (4.38) implies that the left-hand side is unbounded. 


\section{Choice of $a_{k}$}

Let us set

$$
a_{k}^{2}:=\frac{\delta_{k-1}}{k^{2} \eta_{k}^{3}} \exp \left(-2 \varepsilon_{k} \eta_{k} \sqrt{\delta_{k}}\left(s_{k}-t_{k}\right)-32 \varepsilon_{k-1} \eta_{k-1} \sqrt{\delta_{k-1}}\right) .
$$

Conditions (4.35) through (4.38) can thus be restated in terms of the remaining parameters. We obtain the following result (proof is trivial).

Corollary 4.4. Let $v(t)$ be the function defined in (4.32), with $a_{k}$ defined by (4.40).

(1) For every $r>0$ we have that $\left(v(0), v^{\prime}(0)\right) \in \mathcal{G}_{\varphi, r, 3 / 4}(A) \times \mathcal{G}_{\varphi, r, 1 / 4}(A)$ if

$$
\sum_{k=1}^{\infty} \frac{\delta_{k} \delta_{k-1}}{k^{2}} \exp \left(\eta_{k} t_{k}+r \varphi\left(\eta_{k}\right)-2 \varepsilon_{k} \eta_{k} \sqrt{\delta_{k}}\left(s_{k}-t_{k}\right)-32 \varepsilon_{k-1} \eta_{k-1} \sqrt{\delta_{k-1}}\right)<+\infty .
$$

(2) For every $t>0$ and $\beta \geq 0$ we have that $\left(v(t), v^{\prime}(t)\right) \in V_{\beta}$ if

$$
\sum_{k=1}^{\infty} \frac{1}{k^{2}} \eta_{k}^{2 \beta-1}<+\infty
$$

(3) For every $t>0$ and $\beta \geq 0$ we have that $\left(v(t), v^{\prime}(t)\right) \notin V_{\beta}$ if

$$
\sum_{k=1}^{\infty} \frac{\delta_{k} \delta_{k-1}^{2}}{k^{2}} \eta_{k}^{2 \beta-1} \exp \left(-64 \varepsilon_{k-1} \eta_{k-1} \sqrt{\delta_{k-1}}\right)=+\infty .
$$

(4) We have that $v \in C^{1}\left([0,1] ; D\left(A^{1 / 4}\right)\right) \cap C^{0}\left([0,1] ; D\left(A^{3 / 4}\right)\right)$ if (4.41) holds true with $r=0$ (note that (4.42) is trivial for $\beta=1 / 2$ ).

(5) For every $\beta \geq 0$ we have that the sequence $\left|A^{(\beta+1) / 2} v\left(\tau_{k}\right)\right|$ is unbounded if

$$
\lim _{k \rightarrow+\infty} \frac{\delta_{k-1}}{k^{2}} \eta_{k}^{2 \beta-1} \exp \left(2 \varepsilon_{k} \eta_{k} \sqrt{\delta_{k}}\left(\tau_{k}-s_{k}\right)-32 \varepsilon_{k-1} \eta_{k-1} \sqrt{\delta_{k-1}}\right)=+\infty .
$$

\subsection{Proof of Proposition 3.6}

We define $c(t)$ and $v(t)$ according to the general construction. In order to choose the parameters we first set $t_{0}=\delta_{0}=1$. Then for every $k \geq 1$ we set

$$
t_{k}:=\frac{2 \pi}{\eta_{k}}, \quad s_{k}:=\left[\frac{t_{k-1}}{2 t_{k}}\right] t_{k}, \quad \delta_{k}:=1, \quad \varepsilon_{k}:=\omega\left(\frac{1}{\eta_{k}}\right),
$$

where the brackets denote the integer part, and the sequence $\eta_{k}$ is defined as follows: 
- $\eta_{1}$ is any element of the sequence $\left\{\lambda_{k}\right\}$ which is greater than $4 \pi$ and such that $\omega\left(1 / \eta_{1}\right) \leq 1 / 16$

- for every $k \geq 2$ the term $\eta_{k}$ is recursively defined as any element of the sequence $\left\{\lambda_{k}\right\}$ satisfying the following three inequalities

$$
\begin{aligned}
\eta_{k} & \geq 4 \eta_{k-1}, \\
\frac{\eta_{k}}{\varphi\left(\eta_{k}\right)} \omega\left(\frac{1}{\eta_{k}}\right) & \geq \frac{k}{t_{k-1}}, \\
\eta_{k} & \geq \exp \left(k+k \varepsilon_{k-1} \eta_{k-1}\right) .
\end{aligned}
$$

We didn't define $\tau_{k}$ because we don't need it in this proof.

The first thing we have to verify is that $\eta_{k}$ is well defined. The right hand sides of (4.46) through (4.48) only depend on the values of the sequences with index $k-1$. Since the sequence $\left\{\lambda_{k}\right\}$ is unbounded, we can find $\eta_{k}$ with the required properties if we show that the left hand sides tend to $+\infty$ as $\eta_{k} \rightarrow+\infty$. This is trivial in the case of (4.46) and (4.48), and it follows from assumption (3.1) in the case of (4.47).

The second thing we need is that assumptions (Hp- $\left.t_{k}\right),\left(\mathrm{Hp}-s_{k}\right),\left(\mathrm{Hp}-\eta_{k}\right),(\mathrm{Hp}-$ $\delta_{k}$ ), and (Hp- $\left.\varepsilon_{k}\right)$ are satisfied (they are required for the entire construction to work). By (4.46) the sequence $\eta_{k}$ is increasing and tends to $+\infty$. These facts are enough to prove all properties, except the monotonicity of $\varepsilon_{k} \eta_{k} \sqrt{\delta_{k}}$, which however follows from $(\omega 2)$.

We are now ready to prove the conclusions of Proposition 3.6.

\section{Conclusion (SH-1)}

First of all we remark that $1 / 2 \leq c(t) \leq 3 / 2$. This is due to (4.7) and our definition of $\delta_{k}$.

In order to prove the $\omega$-continuity of $c(t)$ we apply Proposition 4.2. The first and second supremum in (4.10) are trivially zero. Moreover by the monotonicity of $\omega$ we have that

$$
\frac{\varepsilon_{k} \delta_{k}}{\omega\left(2 \pi /\left(\eta_{k} \sqrt{\delta_{k}}\right)\right)}=\frac{\varepsilon_{k}}{\omega\left(t_{k}\right)}=\frac{\omega\left(t_{k} /(2 \pi)\right)}{\omega\left(t_{k}\right)} \leq 1,
$$

hence also the third supremum is finite.

\section{Conclusion (SH-2)}

Let us examine (4.41). The series converges for every $r>0$ (and also for $r=0$ ) if we show that the argument of the exponential function is bounded from above. Since $\eta_{k} t_{k}$ and $\varepsilon_{k} \eta_{k} t_{k}$ are bounded, it is enough to prove that $r \varphi\left(\eta_{k}\right) \leq 2 \varepsilon_{k} \eta_{k} s_{k}$ for every large enough $k$. In turn this is true if we show that

$$
\lim _{k \rightarrow+\infty} \frac{\varepsilon_{k} \eta_{k} s_{k}}{\varphi\left(\eta_{k}\right)}=+\infty .
$$


To this end we first use the definition of $t_{k}$ and (4.46) to obtain that

$$
\frac{s_{k}}{t_{k-1}}=\left[\frac{t_{k-1}}{2 t_{k}}\right] \frac{t_{k}}{t_{k-1}} \geq \frac{1}{2}-\frac{t_{k}}{t_{k-1}}=\frac{1}{2}-\frac{\eta_{k-1}}{\eta_{k}} \geq \frac{1}{4} .
$$

Then using the definition of $s_{k}$ and $\varepsilon_{k}$, and estimate (4.47), we deduce that

$$
\frac{\varepsilon_{k} \eta_{k} s_{k}}{\varphi\left(\eta_{k}\right)}=\frac{\eta_{k}}{\varphi\left(\eta_{k}\right)} \omega\left(\frac{1}{\eta_{k}}\right) s_{k} \geq k \cdot \frac{s_{k}}{t_{k-1}} \geq \frac{k}{4},
$$

which implies (4.49).

\section{Conclusion (SH-3)}

By statement (4) of Corollary 4.4 it is enough to prove (4.41) with $r=0$. We did this in the previous paragraph.

\section{Conclusion (SH-4)}

Let us examine (4.43). By (4.48) we have that

$$
\frac{1}{k^{2}} \eta_{k}^{2 \beta-1} \exp \left(-64 \varepsilon_{k-1} \eta_{k-1}\right) \geq \frac{1}{k^{2}} e^{(2 \beta-1) k} \exp \left([(2 \beta-1) k-64] \varepsilon_{k-1} \eta_{k-1}\right) .
$$

It is therefore clear that the series cannot converge if $\beta>1 / 2$.

\subsection{Proof of Proposition 3.7}

Let $g:(0,+\infty) \rightarrow(0,+\infty)$ be defined by $g(\sigma):=\sqrt{\sigma} \omega^{-1}(\sigma)$, where $\omega^{-1}$ is the inverse function of $\omega$. It is easy to see that $g$ is invertible. Its inverse function, which we denote by $h(\sigma)$, is increasing and vanishes as $\sigma \rightarrow 0^{+}$.

Now we define $c(t)$ and $v(t)$ according to the general construction. In order to choose the parameters we first set $t_{0}=\delta_{0}=1$. Then for every $k \geq 1$ we set

$$
\begin{gathered}
t_{k}:=\frac{2 \pi}{\eta_{k} \sqrt{\delta_{k}}}, \quad s_{k}:=\left[\frac{t_{k-1}}{2 t_{k}}\right] t_{k}, \quad \tau_{k}:=s_{k}-\frac{t_{k}}{4}, \\
\varepsilon_{k}:=\frac{1}{16}, \quad \delta_{k}:=h\left(\frac{1}{\eta_{k}}\right),
\end{gathered}
$$

where the brackets denote the integer part, and the sequence $\eta_{k}$ is defined as follows:

- $\eta_{1}$ is any element of the sequence $\left\{\lambda_{k}\right\}$ with $h\left(1 / \eta_{1}\right)<1$, and $\eta_{1} \sqrt{h\left(1 / \eta_{1}\right)}>$ $4 \pi$; 
- for every $k \geq 2$ the term $\eta_{k}$ is recursively defined as any element of the sequence $\left\{\lambda_{k}\right\}$ such that $\eta_{k} \geq \eta_{k-1}+1$ and

$$
\begin{aligned}
\eta_{k}\left[h\left(\frac{1}{\eta_{k}}\right)\right]^{1 / 2} & \geq 4 \eta_{k-1} \sqrt{\delta_{k-1}}, \\
\eta_{k} h\left(\frac{1}{\eta_{k}}\right) & \geq \frac{k}{t_{k-1}}, \\
\frac{\eta_{k}}{\varphi\left(\eta_{k}\right)}\left[h\left(\frac{1}{\eta_{k}}\right)\right]^{1 / 2} & \geq \frac{k}{t_{k-1}}, \\
\eta_{k} h\left(\frac{1}{\eta_{k}}\right) & \geq \frac{k^{2}}{\delta_{k-1}^{2}} \exp \left(64 \varepsilon_{k-1} \eta_{k-1} \sqrt{\delta_{k-1}}\right), \\
\log \eta_{k} & \geq k\left(2 \eta_{k-1} \sqrt{\delta_{k-1}}-\log \delta_{k-1}+2 \log k\right) .
\end{aligned}
$$

The first thing we need is that $\eta_{k}$ is well defined. As in the proof of Proposition 3.6 it is enough to show that the left hand sides of (4.51) through (4.55) tend to $+\infty$ as $\eta_{k} \rightarrow+\infty$. This is trivial in the case of (4.55). As for (4.51), (4.52), (4.54), with the variable change $z=1 / g(\omega(\sigma))$ we have that

$$
\lim _{z \rightarrow+\infty} z\left[h\left(\frac{1}{z}\right)\right]^{\gamma}=\lim _{\sigma \rightarrow 0^{+}} \frac{[\omega(\sigma)]^{\gamma}}{g(\omega(\sigma))}=\lim _{\sigma \rightarrow 0^{+}}[\omega(\sigma)]^{\gamma-3 / 2} \frac{\omega(\sigma)}{\sigma} .
$$

By $(\omega 2)$ the function $\omega(\sigma) / \sigma$ is bounded from below in a right neighborhood of 0 . It follows that the limit is $+\infty$ for every $\gamma<3 / 2$.

Let us finally consider the left hand side of (4.53). Thanks to assumption (3.2) and the variable change $z=1 / g(\omega(1 / \sigma))$ we have that

$$
\begin{aligned}
\lim _{z \rightarrow+\infty} \frac{z}{\varphi(z)}\left[h\left(\frac{1}{z}\right)\right]^{1 / 2} & =\lim _{\sigma \rightarrow+\infty} \frac{1}{g(\omega(1 / \sigma))} \cdot \frac{1}{\varphi(1 / g(\omega(1 / \sigma)))} \cdot[\omega(1 / \sigma)]^{1 / 2} \\
& =\lim _{\sigma \rightarrow+\infty} \sigma\left[\varphi\left(\frac{\sigma}{\sqrt{\omega(1 / \sigma)}}\right)\right]^{-1}=+\infty
\end{aligned}
$$

The second thing we need is that assumptions $\left(\mathrm{Hp}-t_{k}\right),\left(\mathrm{Hp}-s_{k}\right),\left(\mathrm{Hp}-\tau_{k}\right),\left(\mathrm{Hp}-\eta_{k}\right)$, $\left(\mathrm{Hp}-\delta_{k}\right)$, and $\left(\mathrm{Hp}-\varepsilon_{k}\right)$ are satisfied. Assumption (4.51) is equivalent to say that $\eta_{k} \sqrt{\delta_{k}} \geq 4 \eta_{k-1} \sqrt{\delta_{k-1}}$, hence also $t_{k} \leq t_{k-1} / 4$. All the other properties are now almost trivial.

We are now ready to prove the conclusions of Proposition 3.7.

\section{Conclusion (WH-1)}

First of all we remark that $0 \leq c(t) \leq 3 / 2$ (with $c(t)=0$ if and only if $t=0$ ). This is due to (4.6), (4.7), and our definition of $\delta_{k}$. 
In order to prove the $\omega$-continuity of $c(t)$ we apply Proposition 4.2. Let us consider the first supremum in (4.10). By definition of $t_{k}$ and $\delta_{k}$ we have that $\delta_{k}=\omega\left(t_{k} /(2 \pi)\right)$. Since the function $\omega$ is itself $\omega$-continuous, for $j>i$ we have that

$$
\delta_{i}-\delta_{j}=\omega\left(\frac{t_{i}}{2 \pi}\right)-\omega\left(\frac{t_{j}}{2 \pi}\right) \leq \omega\left(\frac{t_{i}-t_{j}}{2 \pi}\right) \leq \omega\left(t_{i}-t_{j}\right),
$$

which implies that the first supremum in (4.10) is finite.

Since clearly $s_{k} / t_{k-1} \leq 1 / 2$, we have that $t_{k-1}-s_{k} \geq t_{k-1} / 2$, hence

$$
\delta_{k-1}-\delta_{k} \leq \delta_{k-1}=\omega\left(\frac{t_{k-1}}{2 \pi}\right) \leq \omega\left(\frac{t_{k-1}}{2}\right) \leq \omega\left(t_{k-1}-s_{k}\right),
$$

which implies that the second supremum in (4.10) is finite. Finally

$$
\delta_{k}=\omega\left(\frac{t_{k}}{2 \pi}\right) \leq \omega\left(t_{k}\right)=\omega\left(\frac{2 \pi}{\eta_{k} \sqrt{\delta_{k}}}\right),
$$

hence also the third supremum is trivially finite.

\section{Conclusion (WH-2)}

Let us examine (4.41). The series converges for every $r>0$ if we show that the argument of the exponential function is bounded from above. Since $\varepsilon_{k} \eta_{k} \sqrt{\delta_{k}} t_{k}$ is constant, it is enough to prove that

$$
\eta_{k} t_{k}+r \varphi\left(\eta_{k}\right) \leq 2 \varepsilon_{k} \eta_{k} \sqrt{\delta_{k}} s_{k}
$$

whenever $k$ is large enough. Since $\varepsilon_{k}$ is constant this is true if we show that

$$
\lim _{k \rightarrow+\infty} \frac{\sqrt{\delta_{k}} s_{k}}{t_{k}}=+\infty, \quad \lim _{k \rightarrow+\infty} \frac{\eta_{k} \sqrt{\delta_{k}} s_{k}}{\varphi\left(\eta_{k}\right)}=+\infty .
$$

Since we already observed that $t_{k} / t_{k-1} \leq 1 / 4$, we have also that

$$
\frac{s_{k}}{t_{k-1}}=\left[\frac{t_{k-1}}{2 t_{k}}\right] \frac{t_{k}}{t_{k-1}} \geq \frac{1}{2}-\frac{t_{k}}{t_{k-1}} \geq \frac{1}{4} .
$$

Combining with (4.52) we obtain that

$$
\frac{\sqrt{\delta_{k}} s_{k}}{t_{k}}=\frac{\delta_{k} \eta_{k} s_{k}}{2 \pi}=\frac{1}{2 \pi} \cdot h\left(\frac{1}{\eta_{k}}\right) \eta_{k} \cdot \frac{s_{k}}{t_{k-1}} \cdot t_{k-1} \geq \frac{k}{8 \pi},
$$

which implies the first limit in (4.56). Using (4.57) and (4.53) we have that

$$
\frac{\eta_{k} \sqrt{\delta_{k}} s_{k}}{\varphi\left(\eta_{k}\right)}=\frac{\eta_{k}}{\varphi\left(\eta_{k}\right)}\left[h\left(\frac{1}{\eta_{k}}\right)\right]^{1 / 2} \frac{s_{k}}{t_{k-1}} \cdot t_{k-1} \geq \frac{k}{4},
$$

which implies the second limit in (4.56). 


\section{Conclusion (WH-3)}

As in the strictly hyperbolic case it follows from the previous paragraph.

\section{Conclusion (WH-4)}

Let us examine (4.43). Assumption (4.54) implies that the general term of the series is greater or equal than $\eta_{k}^{2 \beta-2}$. Therefore the series diverges for every $\beta \geq 1$.

\section{Conclusion (WH-5)}

Let us finally examine (4.44). By (4.55) each term in the sequence is greater or equal than

$$
\exp \left([(2 \beta-1) k-1]\left(2 \eta_{k-1} \sqrt{\delta_{k-1}}-\log \delta_{k-1}+2 \log k\right)-\frac{\pi}{16}\right),
$$

hence its limit is $+\infty$ whenever $\beta>1 / 2$.

\subsection{Proof of Theorem 3.1 and Theorem 3.4}

For shortness's sake we only prove Theorem 3.1 (the proof of Theorem 3.4 is completely analogous).

Let $c(t)$ and $v(t)$ be as in Proposition 3.6. Since properties of $v(t)$ don't depend on a single Fourier component, we can always assume that the component of $v(t)$ with respect to $e_{1}$ is identically zero.

Now we consider the solution $w:[0,1] \rightarrow \mathbb{R}$ of the Cauchy problem

$$
w^{\prime \prime}(t)+\lambda_{1}^{2} c(t) w(t)=0, \quad w(0)=1, w^{\prime}(0)=1,
$$

and for every $\varepsilon>0$ we set

$$
u_{\varepsilon}(t):=w(t) e_{1}+\varepsilon v(t) \quad \forall t \in[0,1] .
$$

The function $u_{\varepsilon}:[0,1] \rightarrow H$ is again a solution of (3.3), and it has the same regularity and non regularity properties of $v(t)$.

Let $\psi_{\varepsilon}(t):=\left|A^{1 / 2} u_{\varepsilon}(t)\right|^{2}$. Since the component of $v(t)$ with respect to $e_{1}$ is zero we have that

$$
\psi_{\varepsilon}^{\prime}(t)=2\left\langle A^{3 / 4} u_{\varepsilon}(t), A^{1 / 4} u_{\varepsilon}^{\prime}(t)\right\rangle=2 \lambda_{1}^{2} w(t) w^{\prime}(t)+2 \varepsilon\left\langle A^{3 / 4} v(t), A^{1 / 4} v^{\prime}(t)\right\rangle .
$$

From (SH-3) we have that $\left\langle A^{3 / 4} v(t), A^{1 / 4} v^{\prime}(t)\right\rangle$ is bounded in $[0,1]$. Since $w(0) w^{\prime}(0)=1$ there exists $T_{0} \in(0,1]$ and $\varepsilon_{0}>0$ such that

$$
\psi_{\varepsilon_{0}}^{\prime}(t) \geq \frac{1}{2} \quad \forall t \in\left[0, T_{0}\right] .
$$


It follows that $\psi_{\varepsilon_{0}}:\left[0, T_{0}\right] \rightarrow\left[\psi_{\varepsilon_{0}}(0), \psi_{\varepsilon_{0}}\left(T_{0}\right)\right]$ is invertible, and therefore we can define $m:[0,+\infty) \rightarrow[1 / 2,3 / 2]$ by

$$
m(\sigma):= \begin{cases}c(0) & \text { if } \sigma \leq \psi_{\varepsilon_{0}}(0) \\ c\left(\psi_{\varepsilon_{0}}^{-1}(\sigma)\right) & \text { if } \psi_{\varepsilon_{0}}(0) \leq \sigma \leq \psi_{\varepsilon_{0}}\left(T_{0}\right), \\ c\left(T_{0}\right) & \text { if } \sigma \geq \psi_{\varepsilon_{0}}\left(T_{0}\right)\end{cases}
$$

The function $m$ is $\omega$-continuous because $\psi_{\varepsilon_{0}}^{-1}$ is Lipschitz continuous. Moreover

$$
c(t)=m\left(\left|A^{1 / 2} u_{\varepsilon_{0}}(t)\right|^{2}\right) \quad \forall t \in\left[0, T_{0}\right] .
$$

Therefore for this choice of the nonlinearity $m$ the function $u(t):=u_{\varepsilon_{0}}(t)$ is a solution of (2.2) in the interval $\left[0, T_{0}\right]$, and it exhibits the required derivative loss.

\section{A. Proof of Theorem A and Theorem B (sketch)}

It is well known that local existence for Kirchhoff equations can be easily proved in different ways (for example Galerkin approximations) provided that solutions are known to satisfy an a priori estimate yielding the uniform continuity of the nonlinear term. In this appendix we prove this estimate under the assumptions of Theorem A and Theorem B. The statement is the following.

Proposition A.1. Let $\omega, m, \varphi, u_{0}, u_{1}, r_{0}$ be as in Theorem A or Theorem B.

Then there exist positive real numbers $T, H, R$, with $R T<r_{0}$, such that every solution

$$
u \in C^{0}\left([0, T] ; D\left(A^{3 / 4}\right)\right) \cap C^{1}\left([0, T] ; D\left(A^{1 / 4}\right)\right)
$$

of problem (2.2), (2.3) satisfies

$$
\left|A^{1 / 4} u^{\prime}(t)\right|^{2}+\left|A^{3 / 4} u(t)\right|^{2} \leq H \quad \forall t \in[0, T],
$$

and

$$
u \in C^{1}\left([0, T] ; \mathcal{G}_{\varphi, r_{0}-R t, 1 / 4}(A)\right) \cap C^{0}\left([0, T] ; \mathcal{G}_{\varphi, r_{0}-R t, 3 / 4}(A)\right) .
$$

The constants $T, H, R$ depend only on $\omega, m$, and on the norms of $u_{0}$ and $u_{1}$ in the spaces $\mathcal{G}_{\varphi, r_{0}, 3 / 4}(A)$ and $\mathcal{G}_{\varphi, r_{0}, 1 / 4}(A)$, respectively.

In the proof of Proposition A.1 we need two simple properties of continuity moduli and convolutions (see [11, Lemma 4.1 and Lemma 4.2]).

Lemma A.2. Let $\omega:[0,+\infty) \rightarrow[0,+\infty)$ be a continuity modulus satisfying $(\omega 1)$.

Then we have that

$$
\begin{gathered}
\omega(\lambda \sigma) \leq(1+\lambda) \omega(\sigma) \quad \forall \lambda \geq 0, \forall \sigma \geq 0 ; \\
1+\frac{1}{\omega(\sigma)} \leq\left(1+\frac{1}{\omega(1)}\right)\left(1+\frac{1}{\sigma}\right) \quad \forall \sigma>0 .
\end{gathered}
$$


Lemma A.3. Let $\rho: \mathbb{R} \rightarrow[0,+\infty)$ be a function of class $C^{\infty}$, with support contained in $[-1,1]$, and integral equal to 1 .

Let $a>0$, and let $f:[0, a] \rightarrow \mathbb{R}$ be a continuous function. Let us extend $f(x)$ to the whole real line by setting $f(x)=f(0)$ for every $x \leq 0$, and $f(x)=f(a)$ for every $x \geq a$.

For every $\varepsilon>0$ let us set

$$
f_{\varepsilon}(x):=\int_{\mathbb{R}} f(x+\varepsilon s) \rho(s) d s \quad \forall x \in \mathbb{R} .
$$

Then $f_{\varepsilon} \in C^{\infty}(\mathbb{R})$, and $f_{\varepsilon}$ has the following properties.

(1) If $\mu_{1} \leq f(x) \leq \mu_{2}$ for every $x \in[0, a]$, then $\mu_{1} \leq f_{\varepsilon}(x) \leq \mu_{2}$ for every $x \in \mathbb{R}$ and every $\varepsilon>0$.

(2) Let $\omega$ be a continuity modulus. Let us assume that

$$
|f(x)-f(y)| \leq H_{0} \omega(|x-y|) \quad \forall x \in[0, a], \forall y \in[0, a],
$$

for some $H_{0} \geq 0$. Then there exists a constant $\gamma_{0}$ (depending on $\rho$, but independent on $\left.\varepsilon, H_{0}, f\right)$ such that

$$
\begin{gathered}
\left|f_{\varepsilon}(x)-f(x)\right| \leq \gamma_{0} H_{0} \omega(\varepsilon) \quad \forall x \in \mathbb{R}, \forall \varepsilon>0, \\
\left|f_{\varepsilon}^{\prime}(x)\right| \leq \gamma_{0} H_{0} \frac{\omega(\varepsilon)}{\varepsilon} \quad \forall x \in \mathbb{R}, \forall \varepsilon>0 .
\end{gathered}
$$

\section{A priori estimates in the strictly hyperbolic case}

Let us introduce some constants. From the assumptions on $m$ and $\varphi$ we know that there exist $L$ and $\Lambda$ such that

$$
\begin{gathered}
\left|m\left(\sigma_{1}\right)-m\left(\sigma_{2}\right)\right| \leq L \omega\left(\left|\sigma_{1}-\sigma_{2}\right|\right) \quad \forall \sigma_{1} \geq 0, \forall \sigma_{2} \geq 0, \\
\sigma \omega(1 / \sigma) \leq \Lambda \varphi(\sigma) \quad \forall \sigma>0 .
\end{gathered}
$$

Let $\gamma_{0}$ be the constant appearing in Lemma A.3, and let

$$
\begin{gathered}
\gamma_{1}:=\left(m\left(\left|A^{1 / 2} u_{0}\right|^{2}\right)+1\right) \cdot \max \left\{1, v^{-1}\right\}, \\
H:=\gamma_{1}\left(\left\|u_{1}\right\|_{\varphi, r_{0}, 1 / 4}^{2}+\left\|u_{0}\right\|_{\varphi, r_{0}, 3 / 4}^{2}\right)+1, \\
R:=\gamma_{0} L \Lambda(H+1)\left(\frac{1}{v}+\frac{1}{\sqrt{v}}\right) .
\end{gathered}
$$

Let $T>0$ be such that

$$
\omega(T) \leq \frac{1}{L(H+1)}, \quad T \leq \frac{r_{0}}{R} .
$$


We claim that (A.2) and (A.3) hold true for these values of $T, H$, and $R$. To this end we introduce

$$
S:=\sup \left\{\tau \leq T:\left|A^{1 / 4} u^{\prime}(t)\right|^{2}+\left|A^{3 / 4} u(t)\right|^{2} \leq H \quad \forall t \in[0, \tau]\right\} .
$$

We have that $S>0$ because $\gamma_{1} \geq 1$ and

$$
\left\|u_{1}\right\|_{D\left(A^{1 / 4}\right)}^{2}+\left\|u_{0}\right\|_{D\left(A^{3 / 4}\right)}^{2} \leq\left\|u_{1}\right\|_{\varphi, r_{0}, 1 / 4}^{2}+\left\|u_{0}\right\|_{\varphi, r_{0}, 3 / 4}^{2}
$$

In order to prove (A.2) we only need to show that $S=T$. So let us assume by contradiction that $S<T$. By the maximality of $S$ we have that necessarily

$$
\left|A^{1 / 4} u^{\prime}(S)\right|^{2}+\left|A^{3 / 4} u(S)\right|^{2}=H .
$$

Moreover for every $t \in[0, S]$ we have that

$$
\left.\left|\frac{\mathrm{d}}{\mathrm{d} t}\right| A^{1 / 2} u(t)\right|^{2}|=2|\left\langle A^{3 / 4} u(t), A^{1 / 4} u^{\prime}(t)\right\rangle \mid \leq H .
$$

Therefore if we set

$$
c(t):=m\left(\left|A^{1 / 2} u(t)\right|^{2}\right),
$$

by (A.7), (A.12), and (A.4) we have that

$$
|c(t)-c(s)| \leq L \omega\left(\left.|| A^{1 / 2} u(t)\right|^{2}-\left|A^{1 / 2} u(s)\right|^{2} \mid\right) \leq L(H+1) \omega(|t-s|)
$$

for every $t$ and $s$ in $[0, S]$. In particular from the strict hyperbolicity and the first inequality in (A.9) we obtain that

$$
v \leq c(t) \leq c(0)+L(H+1) \omega(t) \leq m\left(\left|A^{1 / 2} u_{0}\right|^{2}\right)+1 \quad \forall t \in[0, S] .
$$

Let us extend $c(t)$ outside the interval $[0, S]$ as in Lemma A.3, and let us set

$$
c_{\varepsilon}(t):=\int_{\mathbb{R}} c(t+\varepsilon s) \rho(s) d s \quad \forall t \in \mathbb{R} .
$$

Since estimate (A.6) holds true with $H_{0}:=L(H+1)$, from statements (1) and (2) of Lemma A.3 we deduce that

$$
\begin{aligned}
v \leq c_{\varepsilon}(t) \leq m\left(\left|A^{1 / 2} u_{0}\right|^{2}\right)+1 & \forall t \in \mathbb{R}, \forall \varepsilon>0, \\
\left|c_{\varepsilon}(t)-c(t)\right| \leq \gamma_{0} L(H+1) \omega(\varepsilon) & \forall t \in \mathbb{R}, \forall \varepsilon>0, \\
\left|c_{\varepsilon}^{\prime}(t)\right| \leq \gamma_{0} L(H+1) \frac{\omega(\varepsilon)}{\varepsilon} & \forall t \in \mathbb{R}, \forall \varepsilon>0 .
\end{aligned}
$$

Let us consider the Fourier components $u_{k}(t)$ of $u(t)$, and let us set

$$
E_{k, \varepsilon}(t):=\left|u_{k}^{\prime}(t)\right|^{2}+\lambda_{k}^{2} c_{\varepsilon}(t)\left|u_{k}(t)\right|^{2} .
$$


An easy computation shows that

$$
\begin{aligned}
E_{k, \varepsilon}^{\prime}(t) & =c_{\varepsilon}^{\prime}(t) \lambda_{k}^{2}\left|u_{k}(t)\right|^{2}+2 \lambda_{k}^{2}\left(c_{\varepsilon}(t)-c(t)\right) u_{k}(t) u_{k}^{\prime}(t) \\
& \leq \frac{\left|c_{\varepsilon}^{\prime}(t)\right|}{c_{\varepsilon}(t)} E_{k, \varepsilon}(t)+\lambda_{k} \frac{\left|c_{\varepsilon}(t)-c(t)\right|}{\sqrt{c_{\varepsilon}(t)}} E_{k, \varepsilon}(t),
\end{aligned}
$$

hence by (A.15), (A.16), and (A.17) we obtain that

$$
E_{k, \varepsilon}^{\prime}(t) \leq \gamma_{0} L(H+1)\left(\frac{1}{v} \frac{\omega(\varepsilon)}{\varepsilon}+\frac{1}{\sqrt{v}} \lambda_{k} \omega(\varepsilon)\right) E_{k, \varepsilon}(t) \quad \forall t \in[0, S] .
$$

Let us consider now the eigenvalues $\lambda_{k}>0$, and let us set $\varepsilon_{k}:=1 / \lambda_{k}$. By (A.8) we have that

$$
\frac{\omega\left(\varepsilon_{k}\right)}{\varepsilon_{k}}=\lambda_{k} \omega\left(\varepsilon_{k}\right)=\lambda_{k} \omega\left(\frac{1}{\lambda_{k}}\right) \leq \Lambda \varphi\left(\lambda_{k}\right),
$$

hence by (A.19)

$$
E_{k, \varepsilon_{k}}^{\prime}(t) \leq \gamma_{0} L(H+1)\left(\frac{1}{v}+\frac{1}{\sqrt{v}}\right) \Lambda \varphi\left(\lambda_{k}\right) E_{k, \varepsilon_{k}}(t)=R \varphi\left(\lambda_{k}\right) E_{k, \varepsilon_{k}}(t) .
$$

Integrating this differential inequality we find that

$$
E_{k, \varepsilon_{k}}(t) \leq E_{k, \varepsilon_{k}}(0) \exp \left(R \varphi\left(\lambda_{k}\right) t\right) \quad \forall t \in[0, S]
$$

Combining this inequality with (A.15) we obtain that

$$
\begin{aligned}
\left|u_{k}^{\prime}(t)\right|^{2}+\lambda_{k}^{2}\left|u_{k}(t)\right|^{2} & \leq \max \left\{1, v^{-1}\right\} E_{k, \varepsilon_{k}}(t) \\
& \leq \max \left\{1, v^{-1}\right\} \cdot(c(0)+1)\left(\left|u_{1 k}\right|^{2}+\lambda_{k}^{2}\left|u_{0 k}\right|^{2}\right) \exp \left(\operatorname{Rt} \varphi\left(\lambda_{k}\right)\right) \\
& =\gamma_{1}\left(\left|u_{1 k}\right|^{2}+\lambda_{k}^{2}\left|u_{0 k}\right|^{2}\right) \exp \left(\operatorname{Rt} \varphi\left(\lambda_{k}\right)\right)
\end{aligned}
$$

where $u_{0 k}$ and $u_{1 k}$ denote the Fourier components of $u_{0}$ and $u_{1}$, respectively.

Recalling that $R T \leq r_{0}$, and the definition of $H$, we have that

$$
\begin{aligned}
\left|A^{1 / 4} u^{\prime}(t)\right|^{2}+\left|A^{3 / 4} u(t)\right|^{2} & =\sum_{k} \lambda_{k}\left(\left|u_{k}^{\prime}(t)\right|^{2}+\lambda_{k}^{2}\left|u_{k}(t)\right|^{2}\right) \\
& \leq \gamma_{1} \sum_{k} \lambda_{k}\left(\left|u_{1 k}\right|^{2}+\lambda_{k}^{2}\left|u_{0 k}\right|^{2}\right) \exp \left(r_{0} \varphi\left(\lambda_{k}\right)\right) \\
& =\gamma_{1}\left(\left\|u_{1}\right\|_{\varphi, r_{0}, 1 / 4}^{2}+\left\|u_{0}\right\|_{\varphi, r_{0}, 3 / 4}^{2}\right)<H,
\end{aligned}
$$

for every $t \in[0, S]$. This inequality with $t=S$ contradicts (A.11) and thus proves (A.2). 
A sufficient condition in order to prove (A.3) is that

$$
\mathcal{E}(\tau):=\sum_{k} \lambda_{k} \exp \left(\left(r_{0}-R \tau\right) \varphi\left(\lambda_{k}\right)\right) \sup _{t \in[0, \tau]}\left(\left|u_{k}^{\prime}(t)\right|^{2}+\lambda_{k}^{2}\left|u_{k}(t)\right|^{2}\right)<+\infty
$$

for every $\tau \in(0, T]$. On the other hand from (A.20) we have that

$$
\begin{aligned}
\mathcal{E}(\tau) & \leq \gamma_{1} \sum_{k} \lambda_{k}\left(\left|u_{1 k}\right|^{2}+\lambda_{k}^{2}\left|u_{0 k}\right|^{2}\right) \exp \left(r_{0} \varphi\left(\lambda_{k}\right)\right) \\
& =\gamma_{1}\left(\left\|u_{1}\right\|_{\varphi, r_{0}, 1 / 4}^{2}+\left\|u_{0}\right\|_{\varphi, r_{0}, 3 / 4}^{2}\right) .
\end{aligned}
$$

\section{A priori estimates in the weakly hyperbolic case}

Let $L$ and $\gamma_{0}$ be the constants appearing in (A.7) and in Lemma A.3. Let $\Lambda$ be such that

$$
\sigma \leq \Lambda \varphi\left(\frac{\sigma}{\sqrt{\omega(1 / \sigma)}}\right) \quad \forall \sigma>0
$$

Let us set

$$
\begin{gathered}
\gamma_{2}:=1+\frac{1}{\omega(1)}, \quad \gamma_{3}:=\omega(1)+\max \{\omega(\sigma): 0 \leq \sigma \sqrt{\omega(\sigma)} \leq 1\}, \\
\gamma_{4}:=2 \gamma_{2}\left(\gamma_{3}+m\left(\left|A^{1 / 2} u_{0}\right|^{2}\right)+1\right), \\
H:=\gamma_{4}\left(1+\frac{2 \Lambda}{r_{0}}\right)\left(\left\|u_{1}\right\|_{\varphi, r_{0}, 1 / 4}^{2}+\left\|u_{0}\right\|_{\varphi, r_{0}, 3 / 4}^{2}\right)+1, \\
\gamma_{5}:=\gamma_{0} L(H+1)+1, \quad \gamma_{6}:=2 \gamma_{5} \max \{\Lambda, 1+\sqrt{\omega(1)}\}, \quad R:=2 \gamma_{6} .
\end{gathered}
$$

Let $T>0$ be such that

$$
\omega(T) \leq \frac{1}{L(H+1)}, \quad T \leq \frac{r_{0}}{2 \gamma_{6}} .
$$

We claim that in the weakly hyperbolic case (A.2) and (A.3) hold true with these new values of $T, H$, and $R$. To this end we define once again $S$ according to (A.10), and we assume by contradiction that $S<T$, so that (A.11) holds true.

Let us consider the function $c(t)$ defined according to (A.13), let us extend it outside the interval $[0, S]$ as in Lemma A.3, and let us set

$$
c_{\varepsilon}(t):=\omega(\varepsilon)+\int_{\mathbb{R}} c(t+\varepsilon s) \rho(s) d s \quad \forall t \in \mathbb{R} .
$$

Arguing as in the strictly hyperbolic case we find that

$$
|c(t)-c(s)| \leq L(H+1) \omega(|t-s|)
$$


for every $t$ and $s$ in $[0, S]$. This estimate, together with the first inequality in (A.22), gives that

$$
c(t) \leq c(0)+L(H+1) \omega(t) \leq m\left(\left|A^{1 / 2} u_{0}\right|^{2}\right)+1 \quad \forall t \in[0, S] .
$$

Moreover from statement (2) of Lemma A.3 we deduce that

$$
\begin{aligned}
\left|c_{\varepsilon}(t)-c(t)\right| & \leq\left(1+\gamma_{0} L(H+1)\right) \omega(\varepsilon)=\gamma_{5} \omega(\varepsilon), \\
\left|c_{\varepsilon}^{\prime}(t)\right| & \leq \gamma_{0} L(H+1) \frac{\omega(\varepsilon)}{\varepsilon} \leq \gamma_{5} \frac{\omega(\varepsilon)}{\varepsilon} .
\end{aligned}
$$

Let us consider the Fourier components $u_{k}(t)$ of $u(t)$, and let us define $E_{k, \varepsilon}(t)$ as in (A.18). Computing the time derivative as in the strictly hyperbolic case, and using (A.25), (A.26), and the fact that $c_{\varepsilon}(t) \geq \omega(\varepsilon)$, we find that

$$
E_{k, \varepsilon}^{\prime}(t) \leq \gamma_{5}\left(\frac{1}{\varepsilon}+\lambda_{k} \sqrt{\omega(\varepsilon)}\right) E_{k, \varepsilon}(t) \quad \forall t \in[0, S] .
$$

Now we choose $\varepsilon$ as a function of $k$. If $\lambda_{k}<1$ we set $\varepsilon_{k}=1$, and we have that

$$
\gamma_{5}\left(\frac{1}{\varepsilon_{k}}+\lambda_{k} \sqrt{\omega\left(\varepsilon_{k}\right)}\right) \leq \gamma_{5}(1+\sqrt{\omega(1)}) \leq \gamma_{6} \varphi\left(\lambda_{k}\right),
$$

where we exploited that $\varphi(\sigma) \geq 1$ for all $\sigma \geq 0$.

If $\lambda_{k} \geq 1$ we consider the function $h(\sigma)=\sigma \sqrt{\omega(\sigma)}$, which is invertible, and we set $\varepsilon_{k}:=h^{-1}\left(1 / \lambda_{k}\right)$. By (A.21) we have that

$$
\lambda_{k} \sqrt{\omega\left(\varepsilon_{k}\right)}=\frac{1}{\varepsilon_{k}} \leq \Lambda \varphi\left(\frac{1}{h\left(\varepsilon_{k}\right)}\right)=\Lambda \varphi\left(\lambda_{k}\right)
$$

hence

$$
\gamma_{5}\left(\frac{1}{\varepsilon_{k}}+\lambda_{k} \sqrt{\omega\left(\varepsilon_{k}\right)}\right) \leq \gamma_{6} \varphi\left(\lambda_{k}\right)
$$

Going back to (A.27), in both cases we have that $E_{k, \varepsilon_{k}}^{\prime}(t) \leq \gamma_{6} \varphi\left(\lambda_{k}\right) E_{k, \varepsilon_{k}}(t)$ for every $t \in[0, S]$. Integrating this differential inequality we obtain that

$$
E_{k, \varepsilon_{k}}(t) \leq E_{k, \varepsilon_{k}}(0) \exp \left(\gamma_{6} \varphi\left(\lambda_{k}\right) t\right) \quad \forall t \in[0, S]
$$

In order to estimate $E_{k, \varepsilon_{k}}(0)$ we need an estimate on $c_{\varepsilon_{k}}(0)$. To this end we first observe that

$$
\omega\left(\varepsilon_{k}\right) \leq \gamma_{3} \quad \forall k \in \mathbb{N}
$$

Indeed this estimate is trivial if $\lambda_{k}<1$, while for $\lambda_{k} \geq 1$ it follows from the fact that $h\left(\varepsilon_{k}\right)=1 / \lambda_{k} \leq 1$. Thanks to (A.23), (A.24), and (A.30) we thus obtain that

$$
c_{\varepsilon}(0) \leq \gamma_{3}+m\left(\left|A^{1 / 2} u_{0}\right|^{2}\right)+1
$$


hence

$$
E_{k, \varepsilon_{k}}(0) \leq\left(\gamma_{3}+m\left(\left|A^{1 / 2} u_{0}\right|^{2}\right)+1\right)\left(\left|u_{1 k}\right|^{2}+\lambda_{k}^{2}\left|u_{0 k}\right|^{2}\right)
$$

Moreover from (A.5) and (A.28) it follows that

$$
\max \left\{1, \frac{1}{\omega\left(\varepsilon_{k}\right)}\right\} \leq 1+\frac{1}{\omega\left(\varepsilon_{k}\right)} \leq \gamma_{2}\left(1+\frac{1}{\varepsilon_{k}}\right) \leq 2 \gamma_{2}\left(1+\Lambda \varphi\left(\lambda_{k}\right)\right),
$$

both for $\lambda_{k}<1$ and for $\lambda_{k} \geq 1$. From (A.29), (A.31), and (A.32) it follows that

$$
\begin{aligned}
&\left|u_{k}^{\prime}(t)\right|^{2}+\lambda_{k}^{2}\left|u_{k}(t)\right|^{2} \leq \max \left\{1, \frac{1}{\omega\left(\varepsilon_{k}\right)}\right\} E_{k, \varepsilon_{k}}(t) \\
& \leq \gamma_{4}\left(1+\Lambda \varphi\left(\lambda_{k}\right)\right)\left(\left|u_{1 k}\right|^{2}+\lambda_{k}^{2}\left|u_{0 k}\right|^{2}\right) \exp \left(\gamma_{6} \varphi\left(\lambda_{k}\right) t\right)
\end{aligned}
$$

We are now ready to prove (A.2). We consider the inequality

$$
1+\Lambda x \leq\left(1+\frac{\Lambda}{M}\right) \exp (M x) \quad \forall \Lambda \geq 0, \forall x \geq 0, \forall M>0,
$$

and we apply it with $x=\varphi\left(\lambda_{k}\right), M=r_{0} / 2$. Combined with (A.33) and the fact that $\gamma_{6} T \leq r_{0} / 2$ we obtain that

$$
\left|u_{k}^{\prime}(t)\right|^{2}+\lambda_{k}^{2}\left|u_{k}(t)\right|^{2} \leq \gamma_{4}\left(1+\frac{2 \Lambda}{r_{0}}\right)\left(\left|u_{1 k}\right|^{2}+\lambda_{k}^{2}\left|u_{0 k}\right|^{2}\right) \exp \left(r_{0} \varphi\left(\lambda_{k}\right)\right) .
$$

Multiplying by $\lambda_{k}$, and summing over all $k$ 's, we obtain the same contradiction as in the strictly hyperbolic case.

The proof of (A.3) is quite similar. We fix $\tau \in(0, T]$ and we apply inequality (A.34) with $x=\varphi\left(\lambda_{k}\right), M=\gamma_{6} \tau$. Combined with (A.33) we obtain that

$$
\begin{aligned}
& \exp \left(\left(r_{0}-R \tau\right) \varphi\left(\lambda_{k}\right)\right) \sup _{t \in[0, \tau]}\left(\left|u_{k}^{\prime}(t)\right|^{2}+\lambda_{k}^{2}\left|u_{k}(t)\right|^{2}\right) \leq \\
& \leq \gamma_{4}\left(1+\frac{\Lambda}{\gamma_{6} \tau}\right)\left(\left|u_{1 k}\right|^{2}+\lambda_{k}^{2}\left|u_{0 k}\right|^{2}\right) \exp \left(r_{0} \varphi\left(\lambda_{k}\right)\right)
\end{aligned}
$$

for every $t \in[0, \tau]$. Multiplying by $\lambda_{k}$, and summing over all $k$ 's, we conclude as in the strictly hyperbolic case. 


\section{References}

[1] A. Arosio and S. PANIZZI, On the well-posedness of the Kirchhoff string, Trans. Amer. Math. Soc. 348 (1996), 305-330.

[2] A. Arosio and S. SPAgnolo, Global solutions to the Cauchy problem for a nonlinear hyperbolic equation, In: "Nonlinear Partial Differential Equations and their Applications", Collège de France seminar, Vol. VI (Paris, 1982/1983), Res. Notes in Math., Vol. 109, Pitman, Boston, MA, 1984, 1-26.

[3] S. BERNSTEIN, Sur une classe d'équations fonctionnelles aux dérivées partielles (Russian, French summary), Bull. Acad. Sci. URSS. Sér. Math. [Izvestia Akad. Nauk SSSR] 4 (1940), $17-26$.

[4] F. ColombinI, Quasianalytic and nonquasianalytic solutions for a class of weakly hyperbolic Cauchy problems, J. Differential Equations 241 (2007), 293-304.

[5] F. Colombini, E. De Giorgi and S. Spagnolo, Sur le équations hyperboliques avec des coefficients qui ne dépendent que du temp (French), Ann. Scuola Norm. Sup. Pisa Cl. Sci. (4) 6 (1979), 511-559.

[6] F. Colombini and S. Spagnolo, An example of a weakly hyperbolic Cauchy problem not well posed in $C^{\infty}$, Acta Math. 148 (1982), 243-253.

[7] F. Colombini, E. Jannelli and S. SPAGNolo, Well-posedness in the Gevrey classes of the Cauchy problem for a nonstrictly hyperbolic equation with coefficients depending on time, Ann. Scuola Norm. Sup. Pisa Cl. Sci. (4) 10 (1983), 291-312.

[8] P. D' ANCONA and S. Spagnolo, Global solvability for the degenerate Kirchhoff equation with real analytic data, Invent. Math. 108 (1992), 247-262.

[9] P. D' ANCONA and S. SPAGNOLO, On an abstract weakly hyperbolic equation modelling the nonlinear vibrating string, In: "Developments in Partial Differential Equations and Applications to Mathematical Physics" (Ferrara, 1991), Plenum, New York, 1992, 27-32.

[10] P. D'ANCONA and S. Spagnolo, A class of nonlinear hyperbolic problems with global solutions, Arch. Ration. Mech. Anal. 124 (1993), 201-219.

[11] M. GHISI and M. GobBINO, Spectral gap global solutions for degenerate Kirchhoff equations, Nonlinear Anal. 71 (2009), 4115-4124.

[12] M. GHISI and M. GoBBINO, A uniqueness result for Kirchhoff equations with non-Lipschitz nonlinear term, Adv. Math. (2009), doi:10.1016/j.aim.2009.09.017.

[13] J. M. Greenberg and S. C. HU, The initial value problem for a stretched string, Quart. Appl. Math. 38 (1980/81), 289-311.

[14] F. HIRosawa, Degenerate Kirchhoff equation in ultradifferentiable class, Nonlinear Anal., Ser. A: Theory Methods, 48 (2002), 77-94.

[15] F. HiRos AWA, Global solvability for Kirchhoff equation in special classes of non-analytic functions, J. Differential Equations 230 (2006), 49-70.

[16] G. KirchHoff, "Vorlesungen ober Mathematische Physik: Mechanik", section 29.7, Teubner, Leipzig, 1876.

[17] R. MANFRIN, On the global solvability of Kirchhoff equation for non-analytic initial data, J. Differential Equations 211 (2005), 38-60.

[18] R. MANFRIN, Global solvability to the Kirchhoff equation for a new class of initial data, Port. Math. (N.S.) 59 (2002), 91-109.

[19] K. Nishinara, On a global solution of some quasilinear hyperbolic equation, Tokyo J. Math. 7 (1984), 437-459.

[20] S. I. Pohozaev, On a class of quasilinear hyperbolic equations, Mat. Sb. (N.S.) 96(138) (1975), 152-166) (English transl.: Math. USSR Sbornik 25 (1975), 145-158).

[21] S. I. PoHOzAEv, The Kirchhoff quasilinear hyperbolic equation, Differentsial'nye Uravneniya 21 (1985), 101-108 (English transl.: Differential Equations 21 (1985), 82-87).

[22] M. ReED and B. Simon, "Methods of Modern Mathematical Physics, I: Functional Analysis", Second edition, Academic Press, New York, 1980. 
[23] T. YAMAZAKI, Global solvability for the Kirchhoff equations in exterior domains of dimension three, J. Differential Equations 210 (2005), 290-316.

[24] T. YAMAZAKI, Global solvability for the Kirchhoff equations in exterior domains of dimension larger than three, Math. Methods Appl. Sci. 27 (2004), 1893-1916.

Università degli Studi di Pisa

Dipartimento di Matematica "Leonida Tonelli"

Largo B. Pontecorvo, 5

Pisa, Italia

ghisi@dm.unipi.it

Università degli Studi di Pisa

Dipartimento di Matematica Applicata "Ulisse Dini"

Via Filippo Buonarroti, 1c

56127 Pisa, Italia

m.gobbino@dma.unipi.it 